\title{
Topological mass generation in gapless systems
}

\author{
Naoki Yamamoto \\ Department of Physics, Keio University, Yokohama 223-8522, Japan \\ Ryo Yokokura \\ KEK Theory Center, Tsukuba 305-0801, Japan \\ and Research and Education Center for Natural Sciences, Keio University, Yokohama 223-8521, Japan
}

(Received 11 April 2021; accepted 25 May 2021; published 12 July 2021)

\begin{abstract}
Mass generation of gauge fields can be universally described by topological couplings in gapped systems, such as the Abelian Higgs model in $(3+1)$ dimensions and the Maxwell-Chern-Simons theory in $(2+1)$ dimensions. These systems also exhibit the spontaneous breaking of higher-form $\mathbb{Z}_{k}$ symmetries and topological orders for level $k \geq 2$. In this paper, we consider topological mass generation in gapless systems. As a paradigmatic example, we study the axion electrodynamics with level $k$ in $(3+1)$ dimensions in background fields that hosts both gapped and gapless modes. We argue that the gapped mode is related to those in fully gapped systems in lower dimensions via dimensional reduction. We show that this system exhibits the spontaneous breaking of a higher-form $\mathbb{Z}_{k}$ symmetry despite the absence of the conventional topological order. In the case of the background magnetic field, we also derive the low-energy effective theory of the gapless mode with the quadratic dispersion relation and show that it satisfies the chiral anomaly matching.
\end{abstract}

DOI: 10.1103/PhysRevD.104.025010

\section{INTRODUCTION}

Understanding the origin of the mass is an important question in modern physics. Among others, the Higgs mechanism provides a mechanism to explain the mass generation of gauge fields, such as the massive gauge bosons $W^{ \pm}$and $Z^{0}$ mediating the weak interaction and massive photons in superconductivity. One prototype model of this mechanism is the Abelian Higgs model, where a U(1) gauge field becomes massive by eating a would-be NambuGoldstone (NG) boson. The mass generation can also be described in terms of a low-energy effective theory with the photon and NG boson, called the Stueckelberg theory [1].

However, the Higgs mechanism is not a unique mechanism of the mass generation of gauge fields. In particular, it has been recently shown that photons can acquire a mass gap in $(3+1)$ dimensions even without the conventional Higgs mechanism: in the axion electrodynamics in the presence of background fields, such as a spatially varying axion field [2,3] or an external magnetic field [4,5], one of the helicity states of the photons acquires a mass gap, while the other is gapless with the quadratic dispersion relation.

Published by the American Physical Society under the terms of the Creative Commons Attribution 4.0 International license. Further distribution of this work must maintain attribution to the author(s) and the published article's title, journal citation, and DOI. Funded by SCOAP .
One can ask for a possible universal description explaining both the Higgs mechanism and this helicity-dependent mass generation without the Higgs field.

One such possibility is the mass generation due to a topological coupling of a one-form gauge field and a $(D-2)$-form gauge field in $D$-dimensional spacetime [6]. Here, "topological" means that it does not depend on the metric of the spacetime. Examples in this class of theories include the $B F$ theory [7-9] with kinetic terms in $(3+1)$ dimensions [10,11], Maxwell-Chern-Simons theory in $(2+1)$ dimensions [12-14], and axion electrodynamics in $(1+1)$ dimensions $[15,16]$. In particular, the Stueckelberg theory in $D$ dimensions can be dualized to the one-form and $(D-2)$-form gauge theories with the topological coupling $[6,10]$.

In this paper, we study the axion electrodynamics with level $k$ in $(3+1)$ dimensions ${ }^{1}$ with the background fields above. We argue that the helicity-dependent mass generation of photons in this theory is related to the topological mass generation in gapped systems in lower dimensions via dimensional reduction. What is distinct from the conventional topological mass generation is that this system also hosts gapless modes; hence, this provides an example of "topological mass generation in gapless systems." Since

\footnotetext{
${ }^{1}$ We will refer to the integer appearing in the coefficient of the axion term as the level $k$, similar to the case of the $B F$ theory and Maxwell-Chern-Simons theory.
} 
this system is gapless, there is no conventional topological order that can be seen in gapped systems. Nonetheless, we can show that it exhibits the spontaneous breaking of a higher-form $\mathbb{Z}_{k}$ symmetry similar to the gapped systems with topological order. ${ }^{2}$ Moreover, this system also satisfies the chiral anomaly matching by the gapless modes. In particular, in the case of the external magnetic field, the anomaly matching is satisfied by the gapless mode with the quadratic dispersion relation (in the transverse direction) that may be understood as the so-called type-B NG mode associated with the spontaneous breaking of a one-form symmetry [4] (see also recent discussion [29]).

This paper is organized as follows. In Sec. II, we review the mass generation mechanisms of gauge fields via topological couplings in gapped systems, together with concrete examples, such as the U(1) Abelian Higgs model in $(3+1)$ dimensions, $\mathrm{U}(1)$ Maxwell-Chern-Simons theory in $(2+1)$ dimensions, and axion electrodynamics in $(1+1)$ dimensions. We highlight the spontaneous breaking of higher-form symmetries and its relation to the topological order in each of the examples above. In Sec. III, we study the axion electrodynamics with level $k$ in $(3+1)$ dimensions in background fields. We clarify the relation of the mass generation mechanism in this gapless system to those in gapped systems, the spontaneous breaking of a higher-form $\mathbb{Z}_{k}$ symmetry, and how the chiral anomaly matching is satisfied at low energy. Section IV is devoted to discussions.

Throughout the paper, $a_{\mu}$ denotes a dynamical one-form gauge field, $b_{\mu_{1} \ldots m_{D-2}}$ a dynamical $(D-2)$-form gauge field, $\boldsymbol{e}$ a dynamical electric field, $\boldsymbol{B}$ an external magnetic field, and $\mathcal{A}_{\mu}, \mathcal{B}_{\mu \nu}$ background gauge fields. We use the "mostly plus" metric signature $\eta_{\mu \nu}=\operatorname{diag}(-1,1, \ldots, 1)$ and define the totally antisymmetric tensor $e^{01 \ldots D-1}$ so that $\epsilon^{01 \ldots D-1}=+1$. The $D$-dimensional element on a $D$ dimensional closed subspace, $\mathrm{d} S^{\mu_{1} \cdots \mu_{D}}$, is defined such that it is antisymmetric with respect to the indices, $\mathrm{d} S^{\mu_{1} \cdots \mu_{D}}=$ $\epsilon^{\mu_{1} \cdots \mu_{D}} \mathrm{~d} S_{D}$. We also take the level $k$ to be positive for all the topological couplings without loss of generality.

\section{TOPOLOGICAL MASS GENERATION IN GAPPED SYSTEMS}

In this section, we review the mass generation mechanisms of gauge fields in gapped systems. Although the discussions and results of this section are already known in literature, we include them to make this paper selfcontained, as some of them will be used later in Sec. III

\footnotetext{
${ }^{2}$ Generally, the $p$-form symmetries are symmetries under transformations of $p$-dimensional extended objects [17] (see also Refs. [18-28]). The symmetry generators are $(D-p-1)$ dimensional topological objects. The symmetry transformations are generated by the linking of the symmetry generators and the charged objects.
}

TABLE I. Mass generation of gauge fields via topological couplings in various dimensions. The fields $a_{\mu}, b_{\mu_{1} \ldots \mu_{D-2}}, b_{\mu \nu}, b_{\mu}$, and $\phi$ denote a one-form gauge field, a ( $D-2)$-form gauge field, a two-form gauge field, a one-form gauge field, and a scalar field, respectively. The second column shows the number of massive degrees of freedom (d.o.f.) of $a_{\mu}$ in the presence of the topological coupling. Note that the number of d.o.f. of massless photons in $D$ dimensions is $D-2$.

\begin{tabular}{lcc}
\hline \hline Dimensions & No. of d.o.f. of $a_{\mu}$ & Topological coupling \\
\hline$(D-1)+1$ & $D-1$ & $\epsilon^{\mu_{1} \ldots \mu_{D-2} \nu_{1} \nu_{2}} b_{\mu_{1} \ldots \mu_{D-2}} \partial_{\nu_{1}} a_{\nu_{2}}$ \\
$3+1$ & 3 & $\epsilon^{\mu \nu \rho \sigma} b_{\mu \nu} \partial_{\rho} a_{\sigma}$ \\
$2+1$ & 2 & $\epsilon^{\mu \nu \rho} b_{\mu} \partial_{\nu} a_{\rho}$ \\
$2+1$ & 1 & $\epsilon^{\mu \nu \rho} a_{\mu} \partial_{\nu} a_{\rho}$ \\
$1+1$ & 1 & $\epsilon^{\mu \nu} \phi \partial_{\mu} a_{\nu}$ \\
\hline \hline
\end{tabular}

as a background. The reader may skip to Sec. III and refer back when necessary.

As summarized in Table I, the mass generation mechanism in gapped systems can be described by a topological coupling in arbitrary dimensions [6].

\section{A. U(1) gauge theories with topological couplings}

We first consider the mass generation mechanism by the topological coupling in $D$-dimensional spacetime [6]. We introduce a photon described by a $\mathrm{U}(1)$ one-form gauge field $a_{\mu}$, whose gauge transformation law is $a_{\mu} \rightarrow a_{\mu}+\partial_{\mu} \lambda$ with a $2 \pi$ periodic zero-form gauge parameter $\lambda$.

Here, we assume that the mass dimension of $a_{\mu}$ is one. Generically, a $p$-form is a $p$ th rank antisymmetric tensor. We normalize $a_{\mu}$ by the flux quantization condition $\int_{\mathcal{S}} \frac{1}{2} f_{\mu \nu} \mathrm{d} S^{\mu \nu} \in 2 \pi \mathbb{Z}$, where $f_{\mu \nu}=\partial_{\mu} a_{\nu}-\partial_{\nu} a_{\mu}$ is the field strength of the gauge field, $\mathcal{S}$ is a two-dimensional closed surface without boundaries, and $\mathrm{d} S^{\mu \nu}$ is the area element on $\mathcal{S}$.

In $D$ dimensions, one can couple the one-form gauge field with a $\mathrm{U}(1)(D-2)$-form gauge field $b_{\mu_{1} \ldots \mu_{D-2}}$ by using the totally antisymmetric tensor $\epsilon^{\mu_{1} \ldots \mu_{D}}$. We assume that the mass dimension of $b_{\mu_{1} \ldots \mu_{D-2}}$ is $D-2$. The gauge transformation of $b_{\mu_{1} \ldots \mu_{D-2}}$ is given by

$b_{\mu_{1} \ldots \mu_{D-2}} \rightarrow b_{\mu_{1} \ldots \mu_{D-2}}+\sum_{\sigma \in S_{D-2}} \operatorname{sgn}(\sigma) \partial_{\mu_{\sigma(1)}} \lambda_{\mu_{\sigma(2)} \ldots \mu_{\sigma(D-2)}}$,

where $\lambda_{\mu_{2} \ldots \mu_{D-2}}$ is a $(D-3)$-form parameter, $\sigma$ denotes the permutation of the symmetric group $S_{D-2}$, and $\operatorname{sgn}(\sigma)=$ \pm 1 for even and odd $\sigma$, respectively. The normalization of $b_{\mu_{1} \ldots \mu_{D-2}}$ is $\int_{\Sigma_{D-1}} \frac{1}{(D-1) !} h_{\mu_{1} \ldots \mu_{D-1}} \mathrm{~d} S^{\mu_{1} \cdots \mu_{D-1}} \in 2 \pi \mathbb{Z}$, where $h_{\mu_{1} \ldots \mu_{D-1}}$ is the field strength

$$
h_{\mu_{1} \ldots \mu_{D-1}}=\sum_{\sigma \in S_{D-1}} \operatorname{sgn}(\sigma) \partial_{\mu_{\sigma(1)}} b_{\mu_{\sigma(2)} \ldots \mu_{\sigma(D-1)}},
$$


$\Sigma_{D-1}$ is a $(D-1)$-dimensional closed subspace without boundaries, and $\mathrm{d} S^{\mu_{1} \cdots \mu_{D-1}}$ is the $(D-1)$-dimensional element on $\Sigma_{D-1}$.

We start with the following action:

$$
\begin{aligned}
S_{\text {top }}= & -\int \mathrm{d}^{D} x\left(\frac{1}{8 \pi^{2} v_{D}^{2}(D-1) !}\left|h_{\mu_{1} \ldots \mu_{D-1}}\right|^{2}+\frac{1}{4 e_{D}^{2}}\left|f_{\mu \nu}\right|^{2}\right) \\
& +\frac{k}{2 \pi} \int \mathrm{d}^{D} x \frac{\epsilon^{\mu_{1} \ldots \mu_{D-2} \nu_{1} \nu_{2}}}{(D-2) ! \cdot 2 !} b_{\mu_{1} \ldots \mu_{D-2}} f_{\nu_{1} \nu_{2}}
\end{aligned}
$$

where $v_{D}$ is some constant with mass dimension $\frac{D-2}{2}$ and $e_{D}$ is a coupling constant with mass dimension $\frac{4-D}{2}$. The first line describes the kinetic terms of $a_{\mu}$ and $b_{\mu_{1} \ldots \mu_{D-2}}$, and the second line is the topological coupling. Note that this coupling does not depend on the metric of spacetime since the vector indices are contracted with $\epsilon^{\mu_{1} \ldots \mu_{D}}$, and hence, it is topological. ${ }^{3}$ The topological term is invariant under the gauge transformations of $a_{\mu}$ and $b_{\mu_{1} \ldots \mu_{D-2}}$. The constant $k$ is restricted as $k \in \mathbb{Z}$ by the invariance under large gauge transformations of $a_{\mu}$ and $b_{\mu_{1} \ldots \mu_{D-2}}$.

Since the topological term is quadratic with the firstorder derivative, it generates the masses of $a_{\mu}$ and $b_{\mu_{1} \ldots \mu_{D-2}}$. The equations of motion of $a_{\mu}$ and $b_{\mu_{1} \ldots \mu_{D-2}}$ are

$$
\begin{gathered}
\frac{1}{e_{D}^{2}} \partial_{\nu} f^{\nu \mu}-\frac{k}{2 \pi} \frac{\epsilon^{\nu_{1} \ldots \nu_{D-2} \rho \mu}}{(D-2) !} \partial_{\rho} b_{\nu_{1} \ldots \nu_{D-2}}=0, \\
\frac{1}{4 \pi^{2} v_{D}^{2}} \partial_{\rho} h^{\rho \mu_{1} \ldots \mu_{D-2}}+\frac{k}{2 \pi} \epsilon^{\mu_{1} \ldots \mu_{D-2} \nu \rho} \partial_{\nu} a_{\rho}=0,
\end{gathered}
$$

respectively. By substituting the former into the latter and vice versa, we have

$$
\left(\partial^{2}-\Delta_{D}^{2}\right) f_{\mu \nu}=0, \quad\left(\partial^{2}-\Delta_{D}^{2}\right) h_{\mu_{1} \ldots \mu_{D-2}}=0,
$$

where $\partial^{2} \equiv \partial_{\rho} \partial^{\rho}$ and

$$
\begin{aligned}
& { }^{3} \text { More explicitly, this can be seen as follows. As a generic } \\
& \text { vector field } V_{\mu}(x) \text { transforms under a coordinate transformation } \\
& x \rightarrow x^{\prime}(x) \text { as } V_{\mu}(x)=\frac{\partial x^{\prime \rho}}{\partial x^{\mu}} V_{\rho}^{\prime}\left(x^{\prime}\right) \text {, we have } \\
& \epsilon^{\mu_{1} \ldots \mu_{D-2} \nu_{1} \nu_{2}} b_{\mu_{1} \ldots \mu_{D-2}} f_{\nu_{1} \nu_{2}} \\
& \quad=\epsilon^{\mu_{1} \ldots \mu_{D-2} \nu_{1} \nu_{2}} \frac{\partial x^{\prime \rho_{1}}}{\partial x^{\mu_{1}}} \ldots \frac{\partial x^{\prime \rho_{D-2}}}{\partial x^{\mu_{D-2}}} \frac{\partial x^{\prime \lambda_{1}}}{\partial x^{\nu_{1}}} \frac{\partial x^{\prime \lambda_{2}}}{\partial x^{\nu_{2}}} b_{\rho_{1} \ldots \rho_{D-2}}^{\prime} f_{\lambda_{1} \lambda_{2}}^{\prime} \\
& =\operatorname{det}\left(\frac{\partial x^{\prime}}{\partial x}\right) \epsilon^{\rho_{1} \ldots \rho_{D-2} \lambda_{1} \lambda_{2}} b_{\rho_{1} \ldots \rho_{D-2}}^{\prime} f_{\lambda_{1} \lambda_{2}}^{\prime} .
\end{aligned}
$$

Combined with the relation $\mathrm{d}^{D} x^{\prime}=\operatorname{det}\left(\frac{\partial x^{\prime}}{\partial x}\right) \mathrm{d}^{D} x$, we arrive at

$$
\mathrm{d}^{D} x \epsilon^{\mu_{1} \ldots \mu_{D-2} \nu_{1} \nu_{2}} b_{\mu_{1} \ldots \mu_{D-2}} f_{\nu_{1} \nu_{2}}=\mathrm{d}^{D} x^{\prime} \epsilon^{\rho_{1} \ldots \rho_{D-2} \lambda_{1} \lambda_{2}} b_{\rho_{1} \ldots \rho_{D-2}}^{\prime} f_{\lambda_{1} \lambda_{2}}^{\prime} .
$$

Hence, the last term in Eq. (3) is invariant under general coordinate transformations without the metric.

$$
\Delta_{D}^{2}=\left(k e_{D} v_{D}\right)^{2},
$$

which shows that $f_{\mu \nu}$ and $h_{\mu_{1} \ldots \mu_{D-2}}$ are both massive.

In order to count the physical degrees of freedom, we locally solve the equations of motion in Eqs. (4) and (5) as

$$
\frac{1}{e_{D}^{2}} f^{\rho \mu}-\frac{k}{2 \pi} \frac{\epsilon^{\nu_{1} \ldots \nu_{D-2} \rho \mu}}{(D-2) !}\left[b_{\nu_{1} \ldots \nu_{D-2}}-(D-2) \partial_{\nu_{1}} \bar{b}_{\nu_{2} \ldots \nu_{D-2}}\right]=0 \text {, }
$$

$$
\frac{1}{4 \pi^{2} v_{D}^{2}} h^{\rho \mu_{1} \ldots \mu_{D-2}}+\frac{k}{2 \pi} \epsilon^{\mu_{1} \ldots \mu_{D-2} \rho \sigma}\left(a_{\sigma}-\partial_{\sigma} \bar{a}\right)=0 .
$$

Here, the (D-3)- and zero-form fields $\bar{b}_{\nu_{2} \ldots \nu_{D-2}}$ and $\bar{a}$ are possible ambiguities of the solutions, and they can be absorbed into $b_{\mu_{1} \ldots \mu_{D-2}}$ and $a_{\mu}$ by gauge fixing. Combining the solutions in Eqs. (8) and (9) with the Bianchi identities leads to the constraints on the gauge fields,

$$
\frac{k}{2 \pi} \partial^{\nu_{1}} b_{\nu_{1} \ldots \nu_{D-2}}=0, \quad \frac{k}{2 \pi} \partial^{\sigma} a_{\sigma}=0,
$$

which provide $\frac{(D-1)(D-2)}{2}$ and 1 constraints, respectively. Therefore, both $a_{\mu}$ and $b_{\mu_{1} \ldots \mu_{D-2}}$ have $D-1=\frac{D(D-1)}{2}-$ $\frac{(D-1)(D-2)}{2}$ degrees of freedom. Furthermore, $b_{\mu_{1} \ldots \mu_{D-2}}$ and $a_{\mu}$ are related to each other by the equations of motion, and the physical degrees of freedom of this system is $D-1$.

\section{B. Dual Stueckelberg action}

It is known that the action with the topological coupling in Eq. (3) is dual to the so-called Stueckelberg action [1],

$$
S_{\mathrm{St}}=-\int \mathrm{d}^{D} x\left(\frac{v_{D}^{2}}{2}\left|\partial_{\mu} \chi-k a_{\mu}\right|^{2}+\frac{1}{4 e_{D}^{2}}\left|f_{\mu \nu}\right|^{2}\right),
$$

where $\chi$ is a $2 \pi$ periodic scalar field. The gauge transformation of $\chi$ is $\chi \rightarrow \chi+k \lambda$ with $a_{\mu} \rightarrow a_{\mu}+\partial_{\mu} \lambda$. One can impose the gauge-fixing condition $\chi=0$ (unitary gauge) by using the gauge freedom of $a_{\mu}$. In this gauge, the term $\left|\partial_{\mu} \chi-k a_{\mu}\right|^{2}$ becomes the mass term of $a_{\mu}$.

The Stueckelberg action describes a massive photon with $(D-1)$ polarizations. The equations of motion of $a_{\mu}$ and $\chi$ are given by

$$
\begin{gathered}
v_{D}^{2} k\left(\partial_{\mu} \chi-k a_{\mu}\right)+\frac{1}{e_{D}^{2}} \partial^{\nu} f_{\nu \mu}=0, \\
v_{D}^{2} \partial^{\mu}\left(\partial_{\mu} \chi-k a_{\mu}\right)=0,
\end{gathered}
$$

respectively. Under the gauge condition $\chi=0$, the equation of motion of $a_{\mu}$ reduces to

$$
\left(\partial^{2}-\Delta_{D}^{2}\right) a_{\mu}=0,
$$


with one constraint

$$
k v_{D}^{2} \partial^{\mu} a_{\mu}=0 .
$$

The latter corresponds to the constraint in Eq. (10).

These equations show the presence of massive excitations with three polarizations. More explicitly, for the plane wave ansatz $a_{\mu}=\epsilon_{\mu}(p) \mathrm{e}^{-\mathrm{i} \omega t+\mathrm{i} \cdot \boldsymbol{x}}$ with $p^{\mu}=(\omega, \boldsymbol{p})$ the $D$ momentum, we have

$$
\begin{gathered}
\left(\omega^{2}-\boldsymbol{p}^{2}-\Delta_{D}^{2}\right) \epsilon_{\mu}(p)=0, \\
p^{\mu} \epsilon_{\mu}(p)=0 .
\end{gathered}
$$

Therefore, it has the mass gap, and one of the polarizations, $\epsilon_{0}(p)$, vanishes in the rest frame of the massive gauge field.

We now see that the Stueckelberg action is dual to the action with the topological coupling $[6,10]$. In other words, they are (classically) equivalent to each other. To see this, we introduce the following action:

$$
\begin{aligned}
S_{\mathrm{St}}^{\prime}= & -\int \mathrm{d}^{D} x\left(\frac{v_{D}^{2}}{2}\left|w_{\mu}\right|^{2}+\frac{1}{4 e_{D}^{2}}\left|f_{\mu \nu}\right|^{2}\right) \\
& +\int \mathrm{d}^{D} x \frac{(-1)^{D-2}}{2 \pi} \frac{\epsilon^{\mu_{1} \ldots \mu_{D-1} \nu}}{(D-1) !} h_{\mu_{1} \ldots \mu_{D-1}}\left(w_{\nu}-\partial_{\nu} \chi-k a_{\nu}\right),
\end{aligned}
$$

where $w_{\mu}$ and $h_{\mu_{1} \ldots \mu_{D-1}}$ are one- and (D-1)-form fields, respectively. Using the equation of motion of $h_{\mu_{1} \ldots \mu_{D-1}}$, we can recover the original action (11). Instead, we can dualize the action by eliminating $\chi$. First, the equation of motion of $\chi$ is given by $\epsilon^{\mu_{1} \ldots \mu_{D-1} \nu} \partial_{\nu} h_{\mu_{1} \ldots \mu_{D-1}}=0$. Then, the $(D-1)$-form field $h_{\mu_{1} \ldots \mu_{D-1}}$ can be locally expressed by a $(D-2)$-form gauge field $b_{\mu_{1} \ldots \mu_{D-2}}$ as

$$
h_{\mu_{1} \ldots \mu_{D-1}}=\sum_{\sigma \in S_{D-1}} \operatorname{sgn}(\sigma) \partial_{\mu_{\sigma(1)}} b_{\mu_{\sigma(2)} \ldots \mu_{\sigma(D-1)}} \text {. }
$$

The solution is invariant under the gauge transformation of $b_{\mu_{1} \ldots \mu_{D-2}}$,

$$
b_{\mu_{1} \ldots \mu_{D-2}} \rightarrow b_{\mu_{1} \ldots \mu_{D-2}}+\sum_{\sigma \in S_{D-2}} \operatorname{sgn}(\sigma) \partial_{\mu_{\sigma(1)}} \lambda_{\mu_{\sigma(2)} \ldots \mu_{\sigma(D-2)}},
$$

where $\lambda_{\mu_{2} \ldots \mu_{D-2}}$ is a $(D-3)$-form parameter. Second, the equation of motion of $w_{\mu}$ is

$$
v_{D}^{2} w^{\nu}=\frac{(-1)^{D-2}}{2 \pi} \frac{\epsilon^{\mu_{1} \ldots \mu_{D-1} \nu}}{(D-1) !} h_{\mu_{1} \ldots \mu_{D-1}} .
$$

Substituting these equations into the action (18), we get the action with the topological term in Eq. (3).

\section{Topological order and spontaneous breaking of higher-form $\mathbb{Z}_{k}$ symmetries}

Here, we comment on the fact that the system above with $D \geq 3$ has the so-called topological order [30-32] and that it exhibits the so-called type-B spontaneous symmetry breaking (SSB) [33-36] of $\mathbb{Z}_{k}$ one- and $(D-2)$-form global symmetries [17]. ${ }^{4}$

In order to see the topological nature of the system, we consider the low-energy limit, where the kinetic terms of the one- and $(D-2)$-form gauge fields are negligible. Consequently, the theory can be described by the topological action

$$
S_{B F}=\frac{k}{2 \pi} \int \mathrm{d}^{D} x \frac{\epsilon^{\mu_{1} \ldots \mu_{D-2} \nu_{1} \nu_{2}}}{(D-2) ! \cdot 2 !} b_{\mu_{1} \ldots \mu_{D-2}} f_{\nu_{1} \nu_{2}} .
$$

This is the so-called $B F$ theory $[7,37]$. In this effective theory, there is no local observable since the field strengths of the gauge fields are zero by the equations of motion, $f_{\rho \sigma}=0$ and $h_{\mu_{1} \ldots \mu_{D-1}}=0$. However, there are nonlocal observables, i.e., the Wilson loop and the vortex world volume,

$$
\begin{aligned}
W(\mathcal{C}) & =\mathrm{e}^{\mathrm{i} \int_{\mathcal{C}} a_{\mu} \mathrm{d} x^{\mu}}, \quad \text { and } \\
V\left(\Sigma_{D-2}\right) & =\mathrm{e}^{\mathrm{i} \int_{\Sigma_{D-2}} \frac{1}{(D-2) !} b_{\mu_{1} \ldots \mu_{D-2}} \mathrm{~d} S^{\mu_{1} \cdots \mu_{D-2}}},
\end{aligned}
$$

respectively. Here, $\mathcal{C}$ is a one-dimensional closed loop, $\Sigma_{D-2}$ is a $(D-2)$-dimensional closed subspace without boundaries, and $\mathrm{d} S^{\mu_{1} \cdots \mu_{D-2}}$ is the $(D-2)$-dimensional element on $\Sigma_{D-2}$. Physically, the Wilson loop is a world line of a probe particle with a unit charge and $V\left(\Sigma_{D-2}\right)$ is a world volume of a codimension 2 quantized magnetic vortex, which is a higher-dimensional generalization of Abrikosov-Nielsen-Olesen vortex in $(3+1)$ dimensions [38,39].

The topological excitations can be seen by the correlation function

$$
\begin{aligned}
\left\langle W(\mathcal{C}) V\left(\Sigma_{D-2}\right)\right\rangle & \equiv \mathcal{N} \int \mathcal{D} a_{\mu} \mathcal{D} b_{\nu_{1} \ldots \nu_{D-2}} W(\mathcal{C}) V\left(\Sigma_{D-2}\right) \mathrm{e}^{\mathrm{i} S_{B F}} \\
& =\mathrm{e}^{\frac{2 \pi i}{k} \operatorname{Link}\left(\mathcal{C}, \Sigma_{D-2}\right)}
\end{aligned}
$$

where $\mathcal{N}$ is a normalization factor such that $\langle 1\rangle=1$, and "Link $\left(\mathcal{C}, \Sigma_{D-2}\right)$ " denotes the linking number of $\mathcal{C}$ and $\Sigma_{D-2}$.

Equation (24) can be derived by integrating out the Wilson loop and the vortex world volume [37,40] (see also recent Refs. [41,42]). To integrate out the Wilson loop first, we rewrite the line integral $\int_{\mathcal{C}} a_{\mu} \mathrm{d} x^{\mu}$ by the Stokes theorem as

\footnotetext{
${ }^{4}$ Generally, type-A and type-B SSB are characterized by the conditions that commutation relations of the broken symmetry generators are zero and nonzero, respectively, whichever for continuous and discrete symmetries.
} 


$$
\int_{\mathcal{C}} a_{\mu} \mathrm{d} x^{\mu}=\int_{\partial \mathcal{S}_{C}} a_{\mu} \mathrm{d} x^{\mu}=\int_{\mathcal{S}_{C}} \frac{1}{2} f_{\mu \nu} \mathrm{d} S^{\mu \nu}
$$

Here, $\mathcal{S}_{C}$ is a two-dimensional surface whose boundary is $\mathcal{C}$, $\partial \mathcal{S}_{C}=\mathcal{C}$ with $\partial$ being the boundary operator. We then rewrite the surface integral $\int_{\mathcal{S}_{C}} \frac{1}{2} f_{\mu \nu} \mathrm{d} S^{\mu \nu}$ into the spacetime integral by using the delta function as

$$
\begin{aligned}
\int_{\mathcal{S}_{C}} \frac{1}{2} f_{\mu \nu}(y) \mathrm{d} S^{\mu \nu}(y) & =\int \mathrm{d}^{D} x \int_{\mathcal{S}_{C}} \mathrm{~d} S^{\mu \nu}(y) \frac{1}{2} \delta^{D}(x-y) f_{\mu \nu}(x) \\
& =\int \mathrm{d}^{D} x \frac{1}{2} f_{\mu \nu}(x) J^{\mu \nu}\left(x ; \mathcal{S}_{C}\right)
\end{aligned}
$$

where $J^{\mu \nu}\left(x ; \mathcal{S}_{C}\right)$ is defined by

$$
J^{\mu \nu}\left(x ; \mathcal{S}_{C}\right)=\int_{\mathcal{S}_{C}} \delta^{D}(x-y) \mathrm{d} S^{\mu \nu}(y) .
$$

In the following, we abbreviate $J^{\mu \nu}\left(x ; \mathcal{S}_{C}\right)$ to $J^{\mu \nu}\left(\mathcal{S}_{C}\right)$.

Using Eqs. (25) and (26), the topological action and the line integral in Eq. (24) can be written as

$$
\begin{aligned}
& S_{B F}+\int_{\mathcal{C}} a_{\mu} \mathrm{d} x^{\mu}=\frac{k}{2 \pi} \int \mathrm{d}^{D} x \frac{1}{2 !} \\
& \quad \times\left(\frac{1}{(D-2) !} \epsilon^{\mu_{1} \ldots \mu_{D-2} \nu_{1} \nu_{2}} b_{\mu_{1} \ldots \mu_{D-2}}+\frac{2 \pi}{k} J^{\nu_{1} \nu_{2}}\left(\mathcal{S}_{C}\right)\right) f_{\nu_{1} \nu_{2}} .
\end{aligned}
$$

The Wilson loop can now be integrated out by the redefinition

$$
b_{\mu_{1} \ldots \mu_{D-2}} \rightarrow b_{\mu_{1} \ldots \mu_{D-2}}+\frac{\pi}{k} \epsilon_{\mu_{1} \ldots \mu_{D-2} \nu_{1} \nu_{2}} J^{\nu_{1} \nu_{2}}\left(\mathcal{S}_{C}\right)
$$

Meanwhile, this redefinition transforms the vortex world volume in Eq. (24) as

$$
\begin{aligned}
& \int_{\Sigma_{D-2}} b_{\mu_{1} \ldots \mu_{D-2}} \mathrm{~d} S^{\mu_{1} \cdots \mu_{D-2}} \\
& \rightarrow \int_{\Sigma_{D-2}} b_{\mu_{1} \ldots \mu_{D-2}} \mathrm{~d} S^{\mu_{1} \cdots \mu_{D-2}} \\
& \quad+\int_{\Sigma_{D-2}} \frac{\pi}{k} \epsilon_{\mu_{1} \ldots \mu_{D-2} \nu_{1} \nu_{2}} J^{\nu_{1} \nu_{2}}\left(\mathcal{S}_{C}\right) \mathrm{d} S^{\mu_{1} \cdots \mu_{D-2}}
\end{aligned}
$$

The integral of the last term leads to the intersection number of $\mathcal{S}_{C}$ and $\Sigma_{D_{5}-2}$, which is equal to the linking number of $\mathcal{C}$ and $\Sigma_{D-2}$ :

\footnotetext{
${ }^{5}$ We can relate the linking number to a $D$-dimensional Gauss linking number by the relation of the delta function and Green's function [40].
}

$$
\begin{aligned}
& \int_{\Sigma_{D-2}} \frac{1}{(D-2) ! \cdot 2 !} \epsilon_{\mu_{1} \ldots \mu_{D-2} \nu_{1} \nu_{2}} J^{\nu_{1} \nu_{2}}\left(\mathcal{S}_{C}\right) \mathrm{d} S^{\mu_{1} \cdots \mu_{D-2}} \\
& \quad=\operatorname{Link}\left(\mathcal{C}, \Sigma_{D-2}\right) .
\end{aligned}
$$

Therefore, the correlation function is

$$
\left\langle W(\mathcal{C}) V\left(\Sigma_{D-2}\right)\right\rangle=\mathrm{e}^{\frac{2 \pi \operatorname{Li}}{k} \operatorname{Link}\left(\mathcal{C}, \Sigma_{D-2}\right)}\left\langle V\left(\Sigma_{D-2}\right)\right\rangle .
$$

The vortex world volume can be similarly integrated out. In this procedure, the redefinition of $a_{\mu}$ does not give an additional contribution, since the Wilson loop has been already integrated out. Therefore, we have

$$
\left\langle V\left(\Sigma_{D-2}\right)\right\rangle=1
$$

and we arrive at Eq. (24).

Of course, we can first integrate out the vortex world volume, then integrate out the Wilson loop. In this case, we have

$$
\left\langle W(\mathcal{C}) V\left(\Sigma_{D-2}\right)\right\rangle=\mathrm{e}^{\frac{2 \pi i}{k} \operatorname{Link}\left(\mathcal{C}, \Sigma_{D-2}\right)}\langle W(\mathcal{C})\rangle,
$$

and

$$
\langle W(\mathcal{C})\rangle=1
$$

Therefore, we have the same result as Eq. (24).

The topological feature of the correlation function (24) is that it depends only on the linking number. This is the topologically ordered phase [30-32], which can generally be characterized by the condition that the correlation function of the spatially and temporally extended topological objects have a nonzero fractional phase if they are linked to each other.

The fractional phase due to the linking also leads to the ground-state degeneracy on a compact spatial manifold. To see this, it is convenient to switch to the operator formalism. Let us consider the system on the spacetime with a $(D-1)$ dimensional spatial manifold $M_{D-1}$ with nontrivial topology such that both of the Wilson loop and vortex world volume can topologically wrap subspaces of $M_{D-1}$. One of the simplest choice may be $M_{D-1}=S^{D-2} \times S^{1}$, where $S^{D-2}$ and $S^{1}$ are a $(D-2)$-dimensional sphere and a circle, respectively. Since $V\left(\Sigma_{D-2}\right)$ is a topological object, the action of $V\left(\Sigma_{D-2}\right)$ on the ground state $|\Omega\rangle$ does not change the energy of the system. Therefore, we can choose the ground state as an eigenstate of $V\left(\Sigma_{D-2}\right)$ with the eigenvalue $\mathrm{e}^{\mathrm{i} \theta}$. Meanwhile, the Wilson loop $W(\mathcal{C})$ does not change the energy of the system. Thus, we have another ground state $\left|\Omega^{\prime}\right\rangle=W(\mathcal{C})|\Omega\rangle$. We can show that $\left|\Omega^{\prime}\right\rangle$ and $|\Omega\rangle$ are orthogonal to each other. In fact, the inner product $\left\langle\Omega \mid \Omega^{\prime}\right\rangle$ can be evaluated as

$$
\begin{aligned}
\left\langle\Omega \mid \Omega^{\prime}\right\rangle=\langle\Omega|W(\mathcal{C})| \Omega\rangle & =\left\langle\Omega\left|V^{-1}\left(\Sigma_{D-2}\right) W(\mathcal{C}) V\left(\Sigma_{D-2}\right)\right| \Omega\right\rangle \\
& =\mathrm{e}^{\frac{2 \pi i}{k}}\langle\Omega|W(\mathcal{C})| \Omega\rangle \\
& =\mathrm{e}^{\frac{2 \pi i}{k}}\left\langle\Omega \mid \Omega^{\prime}\right\rangle
\end{aligned}
$$


and so $\left\langle\Omega \mid \Omega^{\prime}\right\rangle=0$ for $k \geq 2$. Here, we used Eq. (34), which, in the operator formalism, leads to the equal-time commutation relation

$$
V^{-1}\left(\Sigma_{D-2}\right) W(\mathcal{C}) V\left(\Sigma_{D-2}\right)=\mathrm{e}^{\frac{2 \pi i}{k}} W(\mathcal{C})
$$

if $\Sigma_{D-2}$ and $\mathcal{C}$ has intersection number on $M_{D-1}$. Since $W(\mathcal{C})^{n}$ $(n=0, \ldots, k-1)$ leads to a different phase, there are $k$ degenerate ground states.

In the viewpoint of global symmetries, the correlation function (24) shows that there are spontaneously broken $\mathbb{Z}_{k}$ one- and $(D-2)$-form global symmetries for $k \geq 2$, and the symmetry breaking pattern is classified as type B. To see this, recall that charged objects under the one- and $(D-2)$ form symmetries are $W(\mathcal{C})$ and $V\left(\Sigma_{D-2}\right)$, respectively. The relations (34) and (32) mean that the symmetry generators of the one- and $(D-2)$-form symmetries are $V\left(\Sigma_{D-2}\right)$ and $W(\mathcal{C})$, respectively. Both of these symmetry transformations are parametrized by the discrete group $\mathbb{Z}_{k}$, since $\left\langle W(\mathcal{C})^{k} V\left(\Sigma_{D-2}\right)\right\rangle=1$ and $\left\langle W(\mathcal{C}) V\left(\Sigma_{D-2}\right)^{k}\right\rangle=1$. Then, the nonzero vacuum expectation values (VEVs) in Eqs. (35) and (33) show that the $\mathbb{Z}_{k}$ one- and $(D-2)$ form symmetries are spontaneously broken. In addition, the correlation function (24) shows that the symmetry breaking pattern is type $\mathrm{B}$, where broken symmetry generators are also charged objects.

It should be remarked that, for the existence of the topologically ordered phase, the condition that the system has a mass gap is essential. If the system is gapless, an infinitesimal deformation of the Wilson loop or vortex world volume gives rise to excited states of the one-form or $(D-2)$-form gauge field even in the low-energy limit. In this case, the states $W(\mathcal{C})|\Omega\rangle$ and $V\left(\Sigma_{D-2}\right)|\Omega\rangle$ cannot be ground states. Then, there is no ground-state degeneracy even if the system is put on a topologically nontrivial spatial manifold, and there is no topological order. In Sec. III, we will consider a gapless system with the type-B SSB, which may not be a topologically ordered phase.

\section{Examples}

Let us now review concrete examples of the mass generation mechanism above.

\section{U(1) Abelian Higgs model in $(3+1)$ dimensions}

The first example is the U(1) Abelian Higgs model in $(3+1)$ dimensions. In the dual theory of the Abelian Higgs model, the mass of the gauge field is generated by a topological coupling with a two-form gauge field $[11,43]$.

We consider a Higgs field $\Phi$ in a U(1) gauge theory. The $\mathrm{U}(1)$ charge of $\Phi$ is $k, \Phi \rightarrow \mathrm{e}^{\mathrm{i} k \lambda} \Phi$ with $a_{\mu} \rightarrow a_{\nu}+\partial_{\mu} \lambda$, where $a_{\mu}$ and $\lambda$ are a U(1) one-form gauge field and a zeroform gauge parameter, respectively. We introduce the action
$S_{\mathrm{AH}}=-\int \mathrm{d}^{4} x\left(\frac{v^{2}}{2}\left|\partial_{\mu} \Phi-\mathrm{i} k a_{\mu} \Phi\right|^{2}+V(|\Phi|)+\frac{1}{4 e^{2}}\left|f_{\mu \nu}\right|^{2}\right)$,

where $V(|\Phi|)$ is a potential of the Higgs field such that the Higgs field develops a nonzero $\operatorname{VEV}\langle\Phi\rangle=\frac{v}{\sqrt{2}}$

Well below the mass of the radial excitation of the Higgs field, the low-energy effective action of this theory can be written by the Stueckelberg action

$S_{\mathrm{AH}, \mathrm{eff}}=-\int \mathrm{d}^{4} x\left(\frac{v^{2}}{2}\left|\partial_{\mu} \chi-k a_{\mu}\right|^{2}+\frac{1}{4 e^{2}}\left|f_{\mu \nu}\right|^{2}\right)$.

In $(3+1)$ dimensions, the Stueckelberg action describes a massive photon with three polarizations.

One can dualize the effective action (39) to a two-form gauge theory. By the same procedure explained in Sec. II B, we arrive at the following action with a topological coupling of the one- and two-form gauge fields:

$$
\begin{aligned}
S_{\mathrm{AH}, \text { dual }}= & \int \mathrm{d}^{4} x\left(-\frac{1}{8 \pi^{2} v^{2} \cdot 3 !}\left|h_{\mu \nu \rho}\right|^{2}-\frac{1}{4 e^{2}}\left|f_{\mu \nu}\right|^{2}\right. \\
& \left.+\frac{k}{8 \pi} \epsilon^{\mu \nu \rho \sigma} b_{\mu \nu} f_{\rho \sigma}\right),
\end{aligned}
$$

where $b_{\mu \nu}$ is the two-form gauge field and $h_{\mu \nu \rho}=\partial_{\mu} b_{\nu \rho}+$ $\partial_{\nu} b_{\rho \mu}+\partial_{\rho} b_{\mu \nu}$ is the field strength. The topological term give rises to the masses of $a_{\mu}$ and $b_{\mu \nu}$.

The phase of the Abelian Higgs theory can be classified by the type-B SSB of $\mathbb{Z}_{k}$ one- and two-form symmetries. Here, the charged objects under the one- and two-form symmetries are the Wilson loop of a probe particle $W(\mathcal{C})$ and the world surface of a quantized magnetic vortex string $V(\mathcal{S})$, with a two-dimensional closed world surface $\mathcal{S}$. This is the Abrikosov-Nielsen-Olesen vortex string $[38,39]$. The generators of the one- and two-form symmetries are $V(\mathcal{S})$ and $W(\mathcal{C})$, respectively. Explicitly, the correlation function of $W(\mathcal{C})$ and $V(\mathcal{S})$ is evaluated by the $B F$ theory as

$$
\begin{aligned}
\langle W(\mathcal{C}) V(\mathcal{S})\rangle & =\mathrm{e}^{\frac{2 \pi i}{k} \operatorname{Lin}(\mathcal{C}, S)}\langle V(\mathcal{S})\rangle \\
& =\mathrm{e}^{\frac{2 \pi i}{k} \operatorname{Link}(\mathcal{C}, S)}\langle W(\mathcal{C})\rangle \\
& =\mathrm{e}^{\frac{2 \pi i}{k} \operatorname{Link}(\mathcal{C}, S)} .
\end{aligned}
$$

The first line means that $W(\mathcal{C})$ and $V(\mathcal{S})$ are the symmetry generator and the charged object under the $\mathbb{Z}_{k}$ two-form symmetry, respectively. The second line means that $V(\mathcal{S})$ and $W(\mathcal{C})$ are the symmetry generator and the charged object under the $\mathbb{Z}_{k}$ one-form symmetry, respectively. The third line shows that both of the $\mathbb{Z}_{k}$ one- and two-form symmetries are broken spontaneously, and the correlation functions of the broken symmetry generators is finite; thus, this can be classified as the type-B SSB. The Higgs phase is 
further classified as a topologically ordered phase [32], since both of the extended charged objects obey the nontrivial braiding statistics.

\section{Topological coupling with two one-form gauge fields}

We then consider the $(2+1)$-dimensional version of the action (3). The action is given by

$$
\begin{aligned}
S_{3 \mathrm{D}}= & -\int \mathrm{d}^{3} x\left(\frac{1}{4 e_{3 \mathrm{D}}^{2}}\left|f_{\mu \nu}\right|^{2}+\frac{1}{16 \pi^{2} v_{3 \mathrm{D}}^{2}}\left|h_{\mu \nu}\right|^{2}\right. \\
& \left.-\frac{k}{2 \pi} \epsilon^{\mu \nu \rho} b_{\mu} \partial_{\nu} a_{\rho}\right),
\end{aligned}
$$

where $e_{3 \mathrm{D}}$ is a coupling constant with mass dimension $1 / 2$ and $v_{3 \mathrm{D}}$ is a constant with mass dimension $-1 / 2$. The action gives one massive photon with two polarizations.

This theory is characterized by the type-B SSB of two $\mathbb{Z}_{k}$ one-form global symmetries. The charged objects are Wilson loops $\mathrm{e}^{\mathrm{i} \int_{\mathcal{C}} a_{\mu} \mathrm{d} x^{\mu}}$ and $\mathrm{e}^{\mathrm{i} \int_{\mathcal{C}} b_{\mu} \mathrm{d} x^{\mu}}$. Again, the correlation function is

$$
\begin{aligned}
\left\langle\mathrm{e}^{\mathrm{i} \int_{\mathcal{C}} a_{\mu} \mathrm{d} x^{\mu}} \mathrm{e}^{\mathrm{i} \int_{\mathcal{C}^{\prime}} b_{\mu} \mathrm{d} x^{\mu}}\right\rangle & =\mathrm{e}^{\frac{2 \pi i}{k} \operatorname{Link}\left(\mathcal{C}, C^{\prime}\right)}\left\langle\mathrm{e}^{\mathrm{i} \int_{\mathcal{C}^{\prime}} b_{\mu} \mathrm{d} x^{\mu}}\right\rangle \\
& =\mathrm{e}^{\frac{2 \pi i}{k} \operatorname{Link}\left(\mathcal{C}, C^{\prime}\right)}\left\langle\mathrm{e}^{\mathrm{i} \int_{\mathcal{C}} a_{\mu} \mathrm{d} x^{\mu}}\right\rangle \\
& =\mathrm{e}^{\frac{2 \pi i}{k} \operatorname{Link}\left(\mathcal{C}, C^{\prime}\right)} .
\end{aligned}
$$

These relations show that the generators of the two $\mathbb{Z}_{k}$ one-form symmetries are $\mathrm{e}^{\mathrm{i} \int_{\mathcal{C}} a_{\mu} \mathrm{d} x^{\mu}}$ and $\mathrm{e}^{\mathrm{i} \int_{\mathcal{C}^{\prime}} b_{\mu} \mathrm{d} x^{\mu}}$, and the charged objects are $\mathrm{e}^{\mathrm{i} \int_{\mathcal{C}^{\prime}} b_{\mu} \mathrm{d} x^{\mu}}$ and $\mathrm{e}^{\mathrm{i} \int_{\mathcal{C}} a_{\mu} \mathrm{d} x^{\mu}}$, respectively. Furthermore, both of them exhibit the type-B SSB, and so it is a topologically ordered phase. Note that this action is dual to the $(2+1)$-dimensional Stueckelberg action

$$
S_{3 \mathrm{D}, \mathrm{St}}=-\int \mathrm{d}^{3} x\left(\frac{v_{3 \mathrm{D}}^{2}}{2}\left|\partial_{\mu} \chi-k a_{\mu}\right|^{2}+\frac{1}{4 e_{3 \mathrm{D}}^{2}}\left|f_{\mu \nu}\right|^{2}\right),
$$

which is the low-energy effective theory of the $(2+1)$ dimensional Abelian Higgs model.

\section{U(1) Maxwell-Chern-Simons theory in $(2+1)$ dimensions}

In the above discussion, we have introduced two independent one-form gauge fields. However, one can introduce a topological term by using a single one-form gauge field in $(2+1)$ dimensions, called the Chern-Simons term. This is a specific feature of the $(2+1)$-dimensional theory.

Consider the Maxwell-Chern-Simons action

$$
S_{\mathrm{MCS}}=\int \mathrm{d}^{3} x\left(-\frac{1}{4 e_{3 \mathrm{D}}^{2}}\left|f_{\mu \nu}\right|^{2}+\frac{k}{4 \pi} \epsilon^{\mu \nu \rho} a_{\mu} \partial_{\nu} a_{\rho}\right) .
$$

The equation of motion of $a_{\mu}$ is

$$
\frac{1}{e_{3 \mathrm{D}}^{2}} \partial_{\nu} f^{\nu \mu}+\frac{k}{2 \pi} \epsilon^{\mu \nu \rho} \partial_{\nu} a_{\rho}=0
$$

From an argument similar to the one used in Sec. II A, the Chern-Simons term generates the mass of the photon as [12-14]

$$
\left(\partial^{2}-\Delta_{3 \mathrm{D}}^{2}\right) f_{\mu \nu}=0, \quad \Delta_{3 \mathrm{D}}^{2}=\left(\frac{k e_{3 \mathrm{D}}^{2}}{2 \pi}\right)^{2} .
$$

By using the plane wave ansatz with Eq. (46), we can show that there is one massive degree of freedom in this theory.

In the low-energy limit, the Maxwell-Chern-Simons theory reduces to the Chern-Simons theory

$$
S_{\mathrm{CS}}=\frac{k}{4 \pi} \int \mathrm{d}^{3} x \epsilon^{\mu \nu \rho} a_{\mu} \partial_{\nu} a_{\rho} .
$$

The observable of the theory is the Wilson loop $W(\mathcal{C})=\mathrm{e}^{\mathrm{i} \int_{\mathcal{C}} a_{\mu} \mathrm{d} x^{\mu}}$. The correlation function of two Wilson loops has a nonzero fractional phase if they are linked to each other,

$$
\left\langle W(\mathcal{C}) W\left(\mathcal{C}^{\prime}\right)\right\rangle=\mathrm{e}^{\frac{2 \pi i}{k} \operatorname{Link}\left(\mathcal{C}, C^{\prime}\right)}\left\langle W\left(\mathcal{C}^{\prime}\right)\right\rangle=\mathrm{e}^{\frac{2 \pi}{k} \operatorname{Link}\left(\mathcal{C}, C^{\prime}\right)} .
$$

Therefore, this theory is again in a topologically ordered phase, characterized by the type-B SSB of $\mathbb{Z}_{k}$ one-form symmetry. In the present case, in particular, the generator of the one-form symmetry is the charged object itself.

\section{Axion electrodynamics in $(1+1)$ dimensions}

The final example is an axion-photon system in $(1+1)$ dimensions, which we will call the axion electrodynamics in $(1+1)$ dimensions in this paper. The higher-form symmetries in this system were studied in the context of its fermionic version, the charge- $k$ Schwinger model, in Refs. [44,45] (see also Ref. [46]). In $(1+1)$ dimensions, a massless photon has no on-shell dynamical degrees of freedom. However, the photon can have a dynamical degree of freedom if it becomes massive, e.g., by a topological coupling with a scalar field $[15,16]$.

We introduce the following action:

$$
\begin{aligned}
S_{2 \mathrm{D}}= & \int \mathrm{d}^{2} x\left(-\frac{1}{4 e_{2 \mathrm{D}}^{2}}\left|f_{\mu \nu}\right|^{2}-\frac{v_{2 \mathrm{D}}^{2}}{2}\left|\partial_{\mu} \phi\right|^{2}+\frac{k}{2 \pi} \epsilon^{\mu \nu} a_{\mu} \partial_{\nu} \phi\right. \\
& \left.+\frac{1}{2 e_{2 \mathrm{D}}^{2}} \partial_{\mu}\left(a_{\nu} f^{\mu \nu}\right)\right)
\end{aligned}
$$

where $\phi$ is a $2 \pi$ periodic scalar field, which is the $(1+1)$ dimensional version of $(D-2)$-form, $e_{2 \mathrm{D}}$ is a coupling constant, and $v_{2 \mathrm{D}}$ is some constant. The third term $\frac{k}{2 \pi} \epsilon^{\mu \nu} a_{\mu} \partial_{\nu} \phi$ is a topological coupling between the photon 
and the scalar field. The last term is a boundary term for the kinetic term of the photon, which is needed to have a consistent energy momentum tensor with the equation of motion of $a_{\mu}$ [47-53], but it will be irrelevant to the following discussion.

The equations of motion of $a_{\mu}$ and $\phi$ are

$$
\begin{aligned}
\frac{1}{e_{2 \mathrm{D}}^{2}} \partial_{\nu} f^{\nu \mu}+\frac{k}{2 \pi} \epsilon^{\mu \nu} \partial_{\nu} \phi & =0, \\
v_{2 \mathrm{D}}^{2} \partial^{\mu} \partial_{\mu} \phi+\frac{k}{2 \pi} \epsilon^{\mu \nu} \partial_{\mu} a_{\nu} & =0,
\end{aligned}
$$

which can be rewritten as

$$
\left(\partial^{2}-\Delta_{2 \mathrm{D}}^{2}\right) f_{\mu \nu}=0, \quad\left(\partial^{2}-\Delta_{2 \mathrm{D}}^{2}\right) \partial_{\nu} \phi=0,
$$

respectively, where

$$
\Delta_{2 \mathrm{D}}^{2}=\left(\frac{k e_{2 \mathrm{D}}}{2 \pi v_{2 \mathrm{D}}}\right)^{2}
$$

The Bianchi identity with the local solutions of Eq. (51) after gauge fixing,

$$
f^{\mu \nu}=\frac{e_{2 \mathrm{D}}^{2} k}{2 \pi} \epsilon^{\mu \nu} \phi, \quad \partial^{\mu} \phi=-\frac{v_{2 \mathrm{D}}^{2} k}{2 \pi} \epsilon^{\mu \nu} a_{\nu},
$$

leads to

$$
\frac{k}{2 \pi} \partial^{\nu} a_{\nu}=0 .
$$

Note that the solution of the equation of motion of $a_{\mu}$ does not lead to any constraint on $\phi$. Therefore, the physical degrees of freedom is one, since $\phi$ is constrained by $f^{\mu \nu}$ in Eq. (51).

The low-energy limit is again described by the topological coupling

$$
S_{2 \mathrm{D}, \text { top }}=\frac{k}{2 \pi} \int \mathrm{d}^{2} x \epsilon^{\mu \nu} a_{\mu} \partial_{\nu} \phi .
$$

The observables are the Wilson loop $W(\mathcal{C})=\mathrm{e}^{\mathrm{i} \int_{\mathcal{C}} a}$ and a two-point object $I\left(\mathcal{P}, \mathcal{P}^{\prime}\right)=\mathrm{e}^{\mathrm{i} \phi(\mathcal{P})-\mathrm{i} \phi\left(\mathcal{P}^{\prime}\right)}$. Of course, a onepoint object can also be an observable. However, it is convenient to use this two-point object, since it can measure the difference of the values of $\phi$ separated by the Wilson loop.

The correlation function is

$$
\left\langle W(\mathcal{C}) I\left(\mathcal{P}, \mathcal{P}^{\prime}\right)\right\rangle=\mathrm{e}^{\frac{2 \pi i}{k} \operatorname{Link}\left(\mathcal{C},\left(\mathcal{P}, \mathcal{P}^{\prime}\right)\right)},
$$

where the linking of the loop $\mathcal{C}$ and the two points $\left(\mathcal{P}, \mathcal{P}^{\prime}\right)$ denotes the configuration where the point $\mathcal{P}$ intersects with $\mathcal{C}$ when $\mathcal{P}$ is continuously moved to $\mathcal{P}^{\prime}$. The phase of this system is classified by the type-B SSB of the $\mathbb{Z}_{k}$ one- and zero-form symmetries [17,19-21,54,55].

\section{TOPOLOGICAL MASS GENERATION IN GAPLESS SYSTEMS}

From now on, we consider the topological mass generation in gapless systems. As such an example, we focus on the axion electrodynamics with level $k$ in $(3+1)$ dimensions in a background magnetic field $[4,5]$ or a spatially varying axion field [2,3]. In these backgrounds, there appear a gapped mode and a gapless mode with the quadratic dispersion relation $\omega \sim p^{2}$ depending on the helicity states. It has also been argued that the latter gapless mode may be understood as the type-B NG mode associated with the spontaneous breaking of a one-form symmetry [4]. We here argue that the helicity-dependent mass generation can be explained from a topological viewpoint.

The action is given by

$$
\begin{aligned}
S_{\mathrm{EM}, \phi}[\phi, a]= & \int \mathrm{d}^{4} x\left(-\frac{1}{4 e^{2}}\left|f_{\mu \nu}\right|^{2}-\frac{v^{2}}{2}\left|\partial_{\mu} \phi\right|^{2}\right. \\
& \left.+\frac{k}{16 \pi^{2}} \phi f_{\mu \nu} \tilde{f}^{\mu \nu}\right),
\end{aligned}
$$

where $\phi$ is a $2 \pi$ periodic pseudoscalar field and $\tilde{f}^{\mu \nu} \equiv \frac{1}{2} \epsilon^{\mu \nu \rho \sigma} f_{\rho \sigma}$. The constant $k$ is again restricted as $k \in \mathbb{Z}$ owing to the invariance under the large gauge transformation of $a_{\mu}$ and the periodicity $\phi \rightarrow \phi+2 \pi$. The last term $\frac{k}{16 \pi^{2}} \phi f_{\mu \nu} \tilde{f}^{\mu \nu}$ is the topological term that does not depend on the metric of spacetime. Unlike the previous examples in gapped systems, this is a cubic interaction term and it does not directly contribute to the dispersion relations of excitations in the system. However, it becomes quadratic in dynamical fields in the presence of a background magnetic field (e.g., $\left\langle f_{12}\right\rangle \neq 0$ ) or a spatially varying axion field (e.g., $\left\langle\partial_{3} \phi\right\rangle \neq 0$ ), which can modify their dispersion relations. We note in passing that the same type of action appears in QCD coupled to QED at low energy, where the role of $\phi$ is played by charge neutral pion $\pi_{0}$ and $|k|=1$.

Without the topological term, this system would have a shift symmetry $\phi \rightarrow \phi+c$ with $c$ being a constant. The Noether current associated with this symmetry is $j_{5}^{\mu}=$ $-2 v^{2} \partial^{\mu} \phi$ that satisfies $\partial_{\mu} j_{5}^{\mu}=0$. Here, we chose the normalization of $j_{5}^{\mu}$ such that, when $|k|=1$, it matches that of the axial current carried by pions in the case of QCD. However, this shift symmetry is broken by the presence of the topological term, and consequently, the conservation law is modified to

$$
\partial_{\mu} j_{5}^{\mu}=\frac{k}{8 \pi^{2}} f_{\mu \nu} \tilde{f}^{\mu \nu}
$$

This can be regarded as the chiral anomaly of this system. 
In the following, we will clarify the mechanism of the helicity-dependent mass generation and the properties of this system in detail.

\section{A. Mass generation and gapless modes}

\section{Background magnetic field}

First, we consider the system with a homogeneous background magnetic field, $\left\langle f_{12}\right\rangle=B_{z}>0$. The equation of motion of $\phi, f_{03}=-e_{z}$ and $\tilde{f}_{12}=\epsilon_{1203} f^{03}=-e_{z}$ at the linearized level read

$$
\begin{gathered}
v^{2} \partial^{\mu} \partial_{\mu} \phi-\frac{k B_{z}}{4 \pi^{2}} e_{z}=0 \\
-\frac{1}{e^{2}} \partial^{\mu} \partial_{\mu} e_{z}+\frac{k B_{z}}{4 \pi^{2}}\left(-\partial_{t}^{2}+\partial_{z}^{2}\right) \phi=0
\end{gathered}
$$

The equations of motion in momentum space can be summarized in the matrix form

$$
\left(\begin{array}{cc}
v^{2}\left(\omega^{2}-\boldsymbol{p}^{2}\right) & -\frac{k B_{z}}{4 \pi^{2}} \\
-\frac{k B_{z}}{4 \pi^{2}}\left(\omega^{2}-p_{z}^{2}\right) & \frac{1}{e^{2}}\left(\omega^{2}-\boldsymbol{p}^{2}\right)
\end{array}\right)\left(\begin{array}{c}
\phi \\
e_{z}
\end{array}\right)=0,
$$

where $p^{\mu}=(\omega, \boldsymbol{p})=\left(\omega, p_{x}, p_{y}, p_{z}\right)$.

One can show that there are one gapless mode with the quadratic dispersion (in the transverse direction with respect to $B_{z}$ ) and one gapped mode, ${ }^{6}$

$$
\begin{aligned}
& \omega_{\text {gapless }}^{2}=p_{z}^{2}+\frac{1}{\Delta_{B}^{2}} \boldsymbol{p}_{\perp}^{4}+\mathcal{O}\left(p^{6}\right), \\
& \omega_{\text {gapped }}^{2}=\Delta_{B}^{2}+2 \boldsymbol{p}_{\perp}^{2}+p_{z}^{2}+\mathcal{O}\left(p^{4}\right),
\end{aligned}
$$

where $\boldsymbol{p}_{\perp}=\left(p_{x}, p_{y}, 0\right)$ and

$$
\Delta_{B}^{2}=\left(\frac{k e B_{z}}{4 \pi^{2} v}\right)^{2}
$$

The corresponding eigenvectors are

$$
\begin{aligned}
& \left(\begin{array}{c}
\phi \\
e_{z}
\end{array}\right) \propto\left(\begin{array}{c}
\frac{\Delta_{B}}{e v}+\mathcal{O}\left(p^{2}\right) \\
\frac{p_{\perp}^{4}}{\Delta_{B}^{2}}-p_{\perp}^{2}+\mathcal{O}\left(p^{6}\right)
\end{array}\right), \\
& \left(\begin{array}{c}
\phi \\
e_{z}
\end{array}\right) \propto\left(\begin{array}{c}
\frac{\Delta_{B}}{e v}+\mathcal{O}\left(p^{2}\right) \\
\Delta_{B}^{2}+p_{\perp}^{2}+\mathcal{O}\left(p^{4}\right)
\end{array}\right),
\end{aligned}
$$

\footnotetext{
${ }^{6}$ The longitudinal component of the dispersion relation for the gapless mode $\omega_{\text {gapless, } \|}^{2}=p_{z}^{2}$ is due to the anomalous charge $n=-\frac{k B_{z}}{4 \pi^{2}} \partial_{z} \phi$ in the presence of the background magnetic field. When one further introduces a background charge such that the local charge neutrality condition is satisfied, then this contribution would vanish $[4,5]$.
}

for the gapless and gapped modes, respectively. The gapless mode with the quadratic dispersion in the transverse direction in Eq. (63) can be understood as the type-B NG mode associated with the spontaneous breaking of the one-form symmetry [4].

We now show that the gapped mode in Eq. (65) is related to the gapped mode in the axion electrodynamics in $(1+1)$ dimensions in Sec. IID 4 via dimensional reduction. A simple way to see this is that the topological term $\epsilon^{\mu \nu 12} \phi f_{\mu \nu}\left\langle f_{12}\right\rangle$ in the present theory in the external magnetic field has the same structure as the topological term $\epsilon^{\mu \nu} \phi f_{\mu \nu}$ in the axion electrodynamics in $(1+1)$ dimensions. To be more concrete, consider the gapped mode in $z$ direction by setting $p^{\mu}=\left(\omega, 0,0, p_{z}\right)$. Then, the eigenstate along the $z$ direction, $e_{z}=\frac{e^{2} k B_{z}}{4 \pi^{2}} \phi$, has the same structure as Eq. (54) but with the additional factor $\frac{B_{z}}{2 \pi}$. This factor, as well as the ratio between the mass gaps of the two theories,

$$
\frac{\Delta_{B}}{\Delta_{2 \mathrm{D}}}=\frac{B_{z}}{2 \pi}
$$

can simply be understood as the Landau degeneracy in the transverse direction with respect to $B_{z}$.

In this way, the mass generation in the axion electrodynamics with the external magnetic field in $(3+1)$ dimensions is related to the one in the axion electrodynamics in $(1+1)$ dimensions. As photons have only one physical degree of freedom in the latter, this argument explains that only one of the two helicity states acquires the mass gap in the former, and hence, this is a helicitydependent mass generation.

\section{Spatially varying axion background}

We can similarly discuss the mass generation of photons in the spatially varying axion background field where $\left\langle\partial_{i} \phi\right\rangle \neq 0$. In this background, the equation of motion of $a_{\mu}$ is

$$
\frac{1}{e^{2}} \partial_{\mu} f^{\mu \nu}-\frac{k}{4 \pi^{2}} \frac{\epsilon^{i \nu \rho \sigma}}{2}\left\langle\partial_{i} \phi\right\rangle f_{\rho \sigma}=0 .
$$

In the following, we assume that the variation of the axion field is positive and homogeneous along the $z$ direction, $\left\langle\partial_{z} \phi\right\rangle>0$. In this case, there is a gapped mode of a linear combination of $e_{x}$ and $e_{y}$ propagating in the $z$ direction $[2,3]$.

The equations of motion of $e_{x}$ and $e_{y}$ in momentum space are summarized as

$\left(\begin{array}{cc}\frac{1}{e^{2}}\left(-\omega^{2}+p_{y}^{2}+p_{z}^{2}\right) & -\frac{1}{e^{2}} p_{x} p_{y}+\frac{\mathrm{i} k}{4 \pi^{2}}\left\langle\partial_{z} \phi\right\rangle \omega \\ -\frac{1}{e^{2}} p_{x} p_{y}-\frac{\mathrm{i} k}{4 \pi^{2}}\left\langle\partial_{z} \phi\right\rangle \omega & \frac{1}{e^{2}}\left(-\omega^{2}+p_{x}^{2}+p_{z}^{2}\right)\end{array}\right)\left(\begin{array}{c}e_{x} \\ e_{y}\end{array}\right)=0$. 
The dispersion relations can be found as

$$
\begin{aligned}
& \omega_{\text {gapless }}^{2}=\frac{1}{\Delta_{\phi}^{2}} \boldsymbol{p}^{2} p_{z}^{2}+\mathcal{O}\left(p^{6}\right), \\
& \omega_{\text {gapped }}^{2}=\Delta_{\phi}^{2}+\boldsymbol{p}_{\perp}^{2}+2 p_{z}^{2}+\mathcal{O}\left(p^{4}\right),
\end{aligned}
$$

where

$$
\Delta_{\phi}^{2}=\left(\frac{k e^{2}}{4 \pi^{2}}\left\langle\partial_{z} \phi\right\rangle\right)^{2}
$$

The corresponding eigenvectors are

$$
\begin{aligned}
& \left(\begin{array}{c}
e_{x} \\
e_{y}
\end{array}\right) \propto\left(\begin{array}{c}
p_{x} p_{y}-\mathrm{i}|\boldsymbol{p}|\left|p_{z}\right|+\mathcal{O}\left(p^{4}\right) \\
p_{y}^{2}+p_{z}^{2}-\frac{1}{\Delta_{\phi}^{2}} \boldsymbol{p}^{2} p_{z}^{2}+\mathcal{O}\left(p^{6}\right)
\end{array}\right), \\
& \left(\begin{array}{c}
e_{x} \\
e_{y}
\end{array}\right) \propto\left(\begin{array}{c}
p_{x} p_{y}-\mathrm{i}\left(\Delta_{\phi}^{2}+\frac{p_{\perp}^{2}+2 p_{z}^{2}}{2}\right)+\mathcal{O}\left(p^{4}\right) \\
-\left(\Delta_{\phi}^{2}+p_{x}^{2}+p_{z}^{2}\right)+\mathcal{O}\left(p^{4}\right)
\end{array}\right)
\end{aligned}
$$

for the gapless and gapped modes, respectively.

This gapped mode is related to the one in the MaxwellChern-Simons theory in $(2+1)$ dimensions in Sec. II D 3. One can see that the topological coupling $\frac{k}{4 \pi^{2}} e^{3 \nu \rho \sigma}\left\langle\partial_{z} \phi\right\rangle$ has the same structure as the topological coupling $\frac{k}{2 \pi} \epsilon^{\nu \rho \sigma}$ in the Maxwell-Chern-Simons theory. For the gapped mode, e.g., along the $y$ direction with the momentum $p^{\mu}=$ $\left(\omega, 0, p_{y}, 0\right)$, Eq. (71) becomes

$$
\Delta_{\phi} e_{x}=\mathrm{i} \omega_{\text {gapped }} e_{y} .
$$

This takes the same form as Eq. (46) with $\mu=y$ and $p_{x}=0$ up to the factor $-\frac{\left\langle\partial_{z} \phi\right\rangle}{2 \pi}$. This factor is reflected in the difference of the gaps between the two theories,

$$
\frac{\Delta_{\phi}}{\Delta_{3 \mathrm{D}}}=\frac{\left\langle\partial_{z} \phi\right\rangle}{2 \pi} .
$$

As photons have only one physical degree of freedom in the Maxwell-Chern-Simons theory in $(2+1)$ dimensions, this argument also explains that only one helicity state of photons acquires the mass gap in the system with the spatially varying axion field in $(3+1)$ dimensions.

\section{B. Spontaneous breaking of higher-form $\mathbb{Z}_{k}$ symmetries}

Here, we discuss higher-form symmetries and their breaking of this theory in the presence of the background magnetic field or the spatially varying axion field.

\section{Higher-form symmetries without background fields}

We first review that the theory has $\mathbb{Z}_{k}$ zero- and oneform symmetries as well as $\mathrm{U}(1)$ one- and two-form symmetries [56]. For simplicity, we first consider the case without background fields and we will discuss the case with background fields later in Sec. III B 3. By using the equations of motion of $\phi$ and $a_{\mu}$ derived from the action (58), we can show that there are conserved currents of electric zero- and one-form symmetries,

$$
\begin{aligned}
& j_{\mathrm{E} \phi}^{\mu}=-v^{2} \partial^{\mu} \phi-\frac{k}{16 \pi^{2}} \epsilon^{\mu \nu \rho \sigma} a_{\nu} f_{\rho \sigma}, \\
& j_{\mathrm{E} a}^{\mu \nu}=-\frac{1}{e^{2}} f^{\mu \nu}+\frac{k}{8 \pi^{2}} \epsilon^{\mu \nu \rho \sigma} \phi f_{\rho \sigma} .
\end{aligned}
$$

In addition, the Bianchi identities for $\phi$ and $a_{\mu}$ give the following conserved currents of magnetic two- and oneform symmetries:

$$
j_{\mathrm{M} \phi}^{\mu \nu \rho}=\frac{1}{2 \pi} \epsilon^{\mu \nu \rho \sigma} \partial_{\sigma} \phi, \quad j_{\mathrm{M} a}^{\mu \nu}=\frac{1}{2 \pi} \epsilon^{\mu \nu \rho \sigma} \partial_{\rho} a_{\sigma},
$$

respectively. Note that the normalizations of the currents are determined by the flux quantization conditions. We can construct topological objects from these currents,

$$
\begin{aligned}
& U_{\mathrm{E} \phi}\left(\mathrm{e}^{\mathrm{i} \alpha_{\mathrm{E} \phi}}, \mathcal{V}\right)=\mathrm{e}^{-\mathrm{i} \alpha_{\mathrm{E} \phi} \int_{\mathcal{V}}^{\epsilon_{\mu \nu \rho \sigma}} 3 ! j_{\mathrm{E} \phi}^{\mu} \mathrm{d} S^{\nu \rho \sigma}}, \\
& U_{\mathrm{E} a}\left(\mathrm{e}^{\mathrm{i} \alpha_{\mathrm{E} a}, \mathcal{S}}\right)=\mathrm{e}^{-\mathrm{i} \alpha_{\mathrm{E} a} \int_{\mathcal{S}} \frac{\epsilon_{\mu \nu \rho \sigma}}{2 ! 2 !} j_{\mathrm{E} a}^{\mu \nu} \mathrm{d} S^{\rho \sigma}}, \\
& U_{\mathrm{M} \phi}\left(\mathrm{e}^{\mathrm{i} \alpha_{\mathrm{M} \phi}, \mathcal{C}}\right)=\mathrm{e}^{-\mathrm{i} \alpha_{\mathrm{M} \phi} \int_{\mathcal{C}}^{\frac{\epsilon_{\mu} \rho \rho \sigma}{3 !} j_{\mathrm{M} \phi}^{\mu \mu \rho}} \mathrm{d} x^{\sigma}}, \\
& U_{\mathrm{M} a}\left(\mathrm{e}^{\mathrm{i} \alpha_{\mathrm{M} a}}, \mathcal{S}\right)=\mathrm{e}^{-\mathrm{i} \alpha_{\mathrm{M} a} \int_{\mathcal{S}} \frac{\epsilon_{\mu \nu \rho \sigma}{ }^{2 ! 2 !}}{\mathrm{M}_{\mathrm{M}} a} \mathrm{~d} S^{\rho \sigma}},
\end{aligned}
$$

where $\mathrm{e}^{\mathrm{i} \alpha_{\mathrm{E} \phi}}, \mathrm{e}^{\mathrm{i} \alpha_{\mathrm{E} a}}, \mathrm{e}^{\mathrm{i} \alpha_{\mathrm{M} \phi}}, \mathrm{e}^{\mathrm{i} \alpha_{\mathrm{M} a}} \in \mathrm{U}(1)$ parametrize the symmetry generators and $\mathcal{V}$ is a three-dimensional closed subspace without boundaries. They are topological since they are invariant under small deformations of $\mathcal{V}, \mathcal{S}$, and $\mathcal{C}$ by the equations of motion or Bianchi identities. We put the minus signs in the prefactors of Eqs. (76)-(79) for convenience.

However, $U_{\mathrm{E} \phi}$ and $U_{\mathrm{E} a}$ are not physical observables for generic $\alpha_{\mathrm{E} \phi}$ and $\alpha_{\mathrm{E} a}$ due to the large gauge transformation of $a_{\mu}$ and the $2 \pi$ periodicity of $\phi$. The invariance under the large gauge transformation and the periodicity requires that the parameters $\mathrm{e}^{\mathrm{i} \alpha_{\mathrm{E} \phi}}$ and $\mathrm{e}^{\mathrm{i} \alpha_{\mathrm{E} a}}$ are constrained as $\mathrm{e}^{\mathrm{i} \alpha_{\mathrm{E} \phi}}, \mathrm{e}^{\mathrm{i} \alpha_{\mathrm{E} a}} \in \mathbb{Z}_{k}$. Note that this argument is similar to the quantization of the Chern-Simons level in $(2+1)$ dimensions [57]. Therefore, the symmetry groups of $U_{\mathrm{E} \phi}$ and $U_{\mathrm{E} a}$ are $\mathbb{Z}_{k}$, while the symmetry groups of $U_{\mathrm{M} \phi}$ and $U_{\mathrm{M} a}$ are $\mathrm{U}(1)$.

Let us explain the detail. For $U_{\mathrm{E} \phi}$, the relevant term is $\mathrm{e}^{-\mathrm{i} k \frac{\alpha_{\mathrm{E} \phi}}{8 \pi^{2}} \int_{\nu} a_{\nu} \partial_{\rho} a_{\sigma} \mathrm{d} S^{\nu \rho \sigma}}$, which is subject to the large gauge transformation. In order to make the integrand manifestly gauge invariant, we introduce a four-dimensional space $X_{4}$ whose boundary is $\mathcal{V}, \partial X_{4}=\mathcal{V}$, to write 


$$
\mathrm{e}^{-\mathrm{i} k \frac{\alpha_{\mathrm{E} \phi}}{8 \pi^{2}} \int_{\nu} a_{\nu} \partial_{\rho} a_{\sigma} \mathrm{d} S^{\nu \rho \sigma}}=\mathrm{e}^{-\mathrm{i} k \frac{\alpha_{\mathrm{E} \phi}}{16 \pi^{2}} \int_{X_{4}} \mathrm{~d}^{4} x f_{\mu \nu} \tilde{f}^{\mu \nu}} .
$$

We require that the term should not depend on the choice of the four-dimensional space. This requirement leads to the constraint on the parameter $\mathrm{e}^{\mathrm{i} \alpha_{\mathrm{E}} \phi}$. By choosing a fourdimensional space $X_{4}^{\prime}$ different from $X_{4}$, such that $\partial X_{4}^{\prime}=\mathcal{V}$, we have

$$
\mathrm{e}^{-\mathrm{i} k \frac{\alpha_{\mathrm{E}} \phi}{8 \pi^{2}} \int_{\nu} a_{\nu} \partial_{\rho} a_{\sigma} \mathrm{d} S^{\nu \rho \sigma}}=\mathrm{e}^{-\mathrm{i} k \frac{\alpha_{\mathrm{E} \phi}}{16 \pi^{2}} \int_{X_{4}} \mathrm{~d}^{4} x f_{\mu \nu} \tilde{f}^{\mu \nu}}=\mathrm{e}^{-\mathrm{i} k \frac{\alpha_{\mathrm{E} \phi}}{16 \pi^{2}} \int_{X_{4}^{\prime}} \mathrm{d}^{4} x f_{\mu \nu} \tilde{f}^{\mu \nu}} \text {. }
$$

Therefore, the integral is independent of the choice of the redundant space if the following condition is satisfied:

$$
\mathrm{e}^{-\mathrm{i} k \frac{\alpha_{\mathrm{E} \phi}}{16 \pi^{2}} \int_{Y_{4}} \mathrm{~d}^{4} x f_{\mu \nu} \tilde{f}^{\mu \nu}}=1
$$

where $Y_{4}=X_{4}-X_{4}^{\prime}$ is a four-dimensional manifold that has no boundary, $\partial Y_{4}=\partial X_{4}-\partial X_{4}^{\prime}=0$, but it can generally have cycles which can be wrapped by the field strength $f_{\mu \nu}$. Since $\int_{Y_{4}} \mathrm{~d}^{4} x f_{\mu \nu} \tilde{f}^{\mu \nu} \in 16 \pi^{2} \mathbb{Z}$ by the flux quantization condition, the parameter $\alpha_{\mathrm{E} \phi}$ is constrained as $\mathrm{e}^{\mathrm{i} \alpha_{\mathrm{E} \phi}} \in \mathbb{Z}_{k}$ in order to satisfy the condition (82).

For $U_{\mathrm{E} a}$, the relevant term is $\mathrm{e}^{\frac{\mathrm{i} k \alpha_{\mathrm{E} a}}{8 \pi^{2}}} \int_{\mathcal{S}} \phi f_{\mu \nu} \mathrm{d} S^{\mu \nu}$, which changes under the transformation $\phi \rightarrow \phi+2 \pi$ as

$$
\mathrm{e}^{\frac{\mathrm{i} k \alpha_{\mathrm{E} a} a}{8 \pi^{2}} \int_{\mathcal{S}} \phi f_{\mu \nu} \mathrm{d} S^{\mu \nu}} \rightarrow \mathrm{e}^{\mathrm{i} k \alpha_{\mathrm{E} a}} \int_{\mathcal{S}} f_{\mu \nu} \mathrm{d} S^{\mu \nu} \mathrm{e}^{\frac{\mathrm{i} k \alpha_{\mathrm{E} a}}{8 \pi^{2}} \int_{\mathcal{S}} \phi f_{\mu \nu} \mathrm{d} S^{\mu \nu}} .
$$

To make it invariant under this transformation, we require

$$
\mathrm{e}^{\mathrm{i} k \alpha_{E} a} \int_{\mathcal{S}} f_{\mu \nu} \mathrm{d} S^{\mu \nu}=1,
$$

which constrains the parameter $\alpha_{\mathrm{E} a}$ as $\mathrm{e}^{\mathrm{i} \alpha_{\mathrm{E} a}} \in \mathbb{Z}_{k}$ by the flux quantization condition $\frac{1}{2 !} \int_{\mathcal{S}} f_{\mu \nu} \mathrm{d} S^{\mu \nu} \in 2 \pi \mathbb{Z}$.

\section{Symmetry transformations}

Next, we consider the charged objects that transform under the extended topological objects:

(i) For the electric $\mathbb{Z}_{k}$ zero-form symmetry generated by $U_{\mathrm{E} \phi}$, the charged object is a particle at a point $\mathcal{P}$ in the spacetime,

$$
I\left(q_{\mathrm{E} \phi}, \mathcal{P}\right)=\mathrm{e}^{\mathrm{i} q_{\mathrm{E} \phi} \phi(\mathcal{P})},
$$

where $q_{\mathrm{E} \phi} \in \mathbb{Z}$ is the charge of the particle. The transformation law is given by the correlation function

$$
\left\langle U_{\mathrm{E} \phi}\left(\mathrm{e}^{\mathrm{i} \alpha_{\mathrm{E} \phi}}, \mathcal{V}\right) I\left(q_{\mathrm{E} \phi}, \mathcal{P}\right)\right\rangle=\mathrm{e}^{\mathrm{i} \alpha_{\mathrm{E} \phi} q_{\mathrm{E} \phi} \operatorname{Link}(\mathcal{V}, P)}\left\langle I\left(q_{\mathrm{E} \phi}, \mathcal{P}\right)\right\rangle .
$$

The derivation of this relation will be given at the end of Sec. III B 2. Since $\mathrm{e}^{\mathrm{i} \alpha_{\mathrm{E} \phi}} \in \mathbb{Z}_{k}$ and $q_{\mathrm{E} \phi} \operatorname{Link}(\mathcal{V}, P) \in \mathbb{Z}$, we have $\mathrm{e}^{\mathrm{i} \alpha_{\mathrm{E} \phi} q_{\mathrm{E} \phi} \operatorname{Link}(\mathcal{V}, P)} \in \mathbb{Z}_{k}$. (ii) For the electric $\mathbb{Z}_{k}$ one-form symmetry generated by $U_{\mathrm{E} a}$, the charged object is the Wilson loop $W\left(q_{\mathrm{E} a}, \mathcal{C}\right)=\mathrm{e}^{\mathrm{i} q_{\mathrm{E} a} \int_{\mathcal{C}} a_{\mu} d x^{\mu}}$. The symmetry transformation is given by

$$
\begin{aligned}
& \left\langleU _ { \mathrm { E } a } \left(\mathrm{e}^{\left.\left.\mathrm{i} \alpha_{\mathrm{E} a}, \mathcal{S}\right) W\left(q_{\mathrm{E} a}, \mathcal{C}\right)\right\rangle}\right.\right. \\
& \quad=\mathrm{e}^{\mathrm{i} \alpha_{\mathrm{E} a} q_{\mathrm{E} a} \operatorname{Link}(\mathcal{S}, C)}\left\langle W\left(q_{\mathrm{E} a}, \mathcal{C}\right)\right\rangle .
\end{aligned}
$$

(iii) For the magnetic U(1) two-form symmetry generated by $U_{\mathrm{M} \phi}$, the charged object is a vortex world surface $V\left(q_{\mathrm{M} \phi}, \mathcal{S}\right)$, which can be characterized by the winding number around a loop $\mathcal{C}$,

$$
\int_{\mathcal{C}} \partial_{\mu} \phi \mathrm{d} x^{\mu}=2 \pi q_{\mathrm{M} \phi} \operatorname{Link}(\mathcal{C}, S) .
$$

The symmetry transformation is

$$
\begin{aligned}
& \left\langle U_{\mathrm{M} \phi}\left(\mathrm{e}^{\mathrm{i} \alpha_{\mathrm{M} \phi}}, \mathcal{C}\right) V\left(q_{\mathrm{M} \phi}, \mathcal{S}\right)\right\rangle \\
& \quad=\mathrm{e}^{\mathrm{i} \alpha_{\mathrm{M} \phi} q_{\mathrm{M} \phi} \operatorname{Link}(\mathcal{C}, S)}\left\langle V\left(q_{\mathrm{M} \phi}, \mathcal{S}\right)\right\rangle .
\end{aligned}
$$

(iv) For the magnetic U(1) one-form symmetry generated by $U_{\mathrm{M} a}$, the charged object is the 't Hooft loop, i.e., a monopole world line $T\left(q_{\mathrm{M} a}, \mathcal{C}\right)$, which can be characterized by the presence of the magnetic flux through a closed surface $\mathcal{S}$ (e.g, a sphere $S^{2}$ ),

$$
\frac{1}{2} \int_{\mathcal{S}} f_{\mu \nu}(x) \mathrm{d} S^{\mu \nu}=2 \pi q_{\mathrm{M} a} \operatorname{Link}(\mathcal{S}, C) .
$$

The symmetry transformation is

$$
\begin{aligned}
& \left\langle U_{\mathrm{M} a}\left(\mathrm{e}^{\mathrm{i} \alpha_{\mathrm{M} a}}, \mathcal{C}\right) T\left(q_{\mathrm{M} a}, \mathcal{C}\right)\right\rangle \\
& \quad=\mathrm{e}^{\mathrm{i} \alpha_{\mathrm{M} a} q_{\mathrm{M} a} \operatorname{Link}(\mathcal{S}, C)}\left\langle T\left(q_{\mathrm{M} a}, \mathcal{C}\right)\right\rangle .
\end{aligned}
$$

Let us derive Eq. (86) as an example. The key relation is the following:

$$
\begin{gathered}
S_{\mathrm{EM}, \phi}[\phi, a]-\alpha_{\mathrm{E} \phi} \int \mathrm{d}^{4} x j_{\mathrm{E} \phi}^{\mu} J_{\mu}(\mathcal{V}) \\
=S_{\mathrm{EM}, \phi}\left[\phi-\alpha_{\mathrm{E} \phi} J\left(\Omega_{\mathcal{V}}\right), a\right]
\end{gathered}
$$

up to the trivial divergence that can be regularized by adding a local counterterm. Here, $\Omega_{\mathcal{V}}$ is a four-dimensional subspace whose boundary is $\mathcal{V}, \partial \Omega_{\mathcal{V}}=\mathcal{V}$, and the symbols $J_{\mu}(\mathcal{V})$ and $J\left(\Omega_{\mathcal{V}}\right)$ are abbreviations of $J_{\mu}(x ; \mathcal{V})$ and $J\left(x ; \Omega_{\mathcal{V}}\right)$, which are defined by

$$
J_{\mu}(x ; \mathcal{V})=\frac{\epsilon_{\mu \nu \rho \sigma}}{3 !} \int_{\mathcal{V}} \delta^{4}(x-y) \mathrm{d} S^{\nu \rho \sigma}(y)
$$

and 


$$
J\left(x ; \Omega_{\mathcal{V}}\right)=\int_{\Omega_{\mathcal{V}}} \mathrm{d}^{4} y \delta^{4}(x-y),
$$

respectively. As in the case of $J^{\mu \nu}\left(\mathcal{S}_{C}\right)$ in Eq. (27), they are introduced such that

$$
\begin{aligned}
\frac{1}{3 !} \int_{\mathcal{V}} \epsilon_{\mu \nu \rho \sigma} j_{\mathrm{E} \phi}^{\mu} \mathrm{d} S^{\nu \rho \sigma} & =\int \mathrm{d}^{4} x j_{\mathrm{E} \phi}^{\mu} J_{\mu}(\mathcal{V}), \\
\int_{\Omega_{\mathcal{V}}} \mathrm{d}^{4} x \partial_{\mu} j_{\mathrm{E} \phi}^{\mu} & =\int \mathrm{d}^{4} x J\left(\Omega_{\mathcal{V}}\right) \partial_{\mu} j_{\mathrm{E} \phi}^{\mu} .
\end{aligned}
$$

In particular, $J\left(\Omega_{\mathcal{V}}\right)$ satisfies

$$
\partial_{\mu} J\left(\Omega_{\mathcal{V}}\right)=J_{\mu}(\mathcal{V}) .
$$

Now, the left-hand side of Eq. (86) can be evaluated by the redefinition $\phi(x)-\alpha_{\mathrm{E} \phi} J\left(\Omega_{\mathcal{V}}\right) \rightarrow \phi(x)$ on the righthand side of Eq. (92) as

$$
\left\langle U_{\mathrm{E} \phi}\left(\mathrm{e}^{\mathrm{i} \alpha_{\mathrm{E} \phi}}, \mathcal{V}\right) I\left(q_{\mathrm{E} \phi}, \mathcal{P}\right)\right\rangle=\mathrm{e}^{\mathrm{i} \alpha_{\mathrm{E} \phi} q_{\mathrm{E} \phi} J\left(\mathcal{P} ; \Omega_{\mathcal{V}}\right)}\left\langle I\left(q_{\mathrm{E} \phi}, \mathcal{P}\right)\right\rangle .
$$

Since $J\left(\mathcal{P} ; \Omega_{\mathcal{V}}\right) \in \mathbb{Z}$ is the intersection number of $\mathcal{P}$ and $\Omega_{\mathcal{V}}$, it counts the linking number $\operatorname{Link}(\mathcal{V}, P)$. Therefore, we obtain the relation (86).

\section{Higher-form symmetries with background fields}

We can describe the external magnetic field and spatially varying axion field in terms of background gauge fields coupled to the symmetry generators of the electric zero- and one-form symmetries. For this purpose, we introduce one- and two-form gauge fields $\mathcal{A}_{\mu}$ and $\mathcal{B}_{\mu \nu}$. At the linear order of $\mathcal{A}_{\mu}$ and $\mathcal{B}_{\mu \nu}$, we can gauge the action (58) by adding the coupling terms $\int \mathrm{d}^{4} x j_{\mathrm{E} \phi}^{\mu} \mathcal{A}_{\mu}$ and $\frac{1}{2} \int \mathrm{d}^{4} x j_{\mathrm{E} a}^{\mu \nu} \mathcal{B}_{\mu \nu}$. However, the invariance under the large gauge transformation of $a_{\mu}$ and the periodicity $\phi \rightarrow \phi+2 \pi$ requires that the background gauge fields should be flat connections satisfying

$$
k \mathcal{A}_{\mu}=\partial_{\mu} \overline{\mathcal{A}} \quad \text { and } \quad k \mathcal{B}_{\mu \nu}=\partial_{\mu} \overline{\mathcal{B}}_{\nu}-\partial_{\nu} \overline{\mathcal{B}}_{\mu}
$$

respectively. Here, $\overline{\mathcal{A}}$ and $\overline{\mathcal{B}}_{\mu}$ are zero- and one-form gauge fields that are normalized by the flux quantization conditions, $\int_{\mathcal{C}} \partial_{\mu} \overline{\mathcal{A}} \mathrm{d} x^{\mu} \in 2 \pi \mathbb{Z}$ and $\int_{\mathcal{S}} \partial_{\mu} \overline{\mathcal{B}}_{\nu} \mathrm{d} S^{\mu \nu} \in 2 \pi \mathbb{Z}$. The couplings of the currents to the background gauge fields are invariant under the gauge transformations

$$
\mathcal{A}_{\mu} \rightarrow \mathcal{A}_{\mu}+\partial_{\mu} \Lambda, \quad \overline{\mathcal{A}} \rightarrow \overline{\mathcal{A}}+k \Lambda,
$$

and

$$
\mathcal{B}_{\mu \nu} \rightarrow \mathcal{B}_{\mu \nu}+\partial_{\mu} \Lambda_{\nu}-\partial_{\nu} \Lambda_{\mu}, \quad \overline{\mathcal{B}}_{\mu} \rightarrow \overline{\mathcal{B}}_{\mu}+k \Lambda_{\mu} .
$$

Here, $\Lambda$ and $\Lambda_{\mu}$ are zero- and one-form gauge parameters normalized as $\int_{\mathcal{C}} \partial_{\mu} \Lambda \mathrm{d} x^{\mu} \in 2 \pi \mathbb{Z}$ and $\int_{\mathcal{S}} \partial_{\mu} \Lambda_{\nu} \mathrm{d} S^{\mu \nu} \in 2 \pi \mathbb{Z}$, respectively.

Now, let us specify the background gauge field that describes the spatially varying axion field or external magnetic field. For the spatially varying axion field, we choose $\mathcal{A}_{\mu}=\delta_{\mu}^{z}\left\langle\partial_{z} \phi(z)\right\rangle$. This choice corresponds to $\overline{\mathcal{A}}=k\langle\phi(z)\rangle$ satisfying $\int_{\mathcal{C}} \partial_{\mu} \overline{\mathcal{A}} \mathrm{d} x^{\mu}=0$. The coupling term $\int \mathrm{d}^{4} x j_{\mathrm{E} \phi}^{\mu} \mathcal{A}_{\mu}$ leads to the desired coupling

$$
\frac{k}{8 \pi^{2}} \int \mathrm{d}^{4} x \epsilon^{3 \mu \nu \rho}\left\langle\partial_{z} \phi(z)\right\rangle a_{\mu} \partial_{\nu} a_{\rho} .
$$

Similarly, the external magnetic field can be realized by choosing $\mathcal{B}_{\mu \nu}=\left(\delta_{\mu}^{x} \delta_{\nu}^{y}-\delta_{\mu}^{y} \delta_{\nu}^{x}\right) B_{z}$ with $\overline{\mathcal{B}}_{\nu}=\frac{1}{2} k\left(x \delta_{\nu}^{y}-y \delta_{\nu}^{x}\right) B_{z}$ satisfying $\int_{\mathcal{S}} \partial_{\mu} \overline{\mathcal{B}}_{\nu} \mathrm{d} S^{\mu \nu}=0$. The coupling term $\frac{1}{2} \int \mathrm{d}^{4} x j_{\mathrm{E} a}^{\mu \nu} \mathcal{B}_{\mu \nu}$ yields

$$
\frac{k}{4 \pi^{2}} \int \mathrm{d}^{4} x \phi f_{03} B_{z} .
$$

We can also couple the system to $\mathcal{A}_{\mu}$ and $\mathcal{B}_{\mu \nu}$ at the nonlinear order. These couplings can be added by the replacements $\partial_{\mu} \phi \rightarrow \partial_{\mu} \phi-\mathcal{A}_{\mu}$ and $f_{\mu \nu} \rightarrow f_{\mu \nu}-\mathcal{B}_{\mu \nu}$ in Eq. (58), respectively. The dynamical fields transform under the gauge transformations in Eqs. (99) and (100) as

$$
\phi \rightarrow \phi+\Lambda,
$$

and

$$
a_{\mu} \rightarrow a_{\mu}+\Lambda_{\mu},
$$

respectively. ${ }^{7}$ The spatially varying axion field and external magnetic field can also be realized by the above choices.

In the gauged action, the magnetic one- and two-form symmetries can be explicitly broken, in general. The gauge invariance under Eqs. (99), (100), (103), and (104) requires the symmetry generators should be deformed as $\mathrm{e}^{\frac{\mathrm{i} \alpha_{\mathrm{M} a} a}{4 \pi} \int_{\mathcal{S}}\left(f_{\mu \nu}-\mathcal{B}_{\mu \nu}\right) \mathrm{d} S^{\mu \nu}}$ for the magnetic one-form symmetry and $\mathrm{e}^{\frac{\mathrm{i} \alpha_{\mathrm{M} \phi}}{2 \pi} \int_{\mathcal{C}}\left(\partial_{\mu} \phi-\mathcal{A}_{\mu}\right) \mathrm{d} x^{\mu}}$ for the magnetic two-form symmetry. However, the presence of the background fields may violate the $2 \pi$ periodicity of the parameters $\alpha_{\mathrm{M} a}$ and $\alpha_{\mathrm{M} \phi}$ by fractional phases due to the relations $\frac{1}{2} \int_{\mathcal{S}} \mathcal{B}_{\mu \nu} \mathrm{d} S^{\mu \nu} \in \frac{2 \pi}{k} \mathbb{Z}$ and $\int_{\mathcal{C}} \mathcal{A}_{\mu} \mathrm{d} x^{\mu} \in \frac{2 \pi}{k} \mathbb{Z}$. In the case of the spatially varying axion field or the external magnetic field, these symmetries are preserved since the background fields do not lead to fractional phases.

\footnotetext{
${ }^{7}$ Technically, the nonlinear gauging of the electric one-form symmetry violates the periodicity $\phi \rightarrow \phi+2 \pi$, and we should gauge the two-form symmetry simultaneously [58]. However, such a violation is absent if we focus on the external magnetic field or the spatially varying axion field discussed in this paper.
} 


\section{Higher-form symmetry breaking}

Here, we explain the higher-form symmetry breaking in the presence of the external fields. In this system, the $\mathbb{Z}_{k}$ zero- and one-form symmetries and U(1) one-form symmetry can be spontaneously broken. ${ }^{8}$

We are interested in the breaking of the electric one-form symmetry characterized by a nonzero VEV of the Wilson loop. Consider a pair of static charges with opposite signs. We assume that the distance of the charges is $R$ and they are created at $t=0$ and annihilated at $t=T$. Under this assumption, the VEV of the Wilson loop is

$$
\left\langle W\left(q_{\mathrm{E} a}, \mathcal{C}\right)\right\rangle=\mathrm{e}^{-T V(R)},
$$

where $V(R)$ is the potential energy. To evaluate $V(R)$, we consider the propagator of the gapless modes in the static limit $\omega=0$.

Let us first consider the case with the background magnetic field. To see whether the symmetry breaking occurs, we look at the kinetic term of $\phi$ and $a_{\mu}$,

$$
\mathcal{L}_{\text {kin }}=\frac{1}{2}\left(\begin{array}{cc}
v \phi & \frac{1}{e} a^{\mu}
\end{array}\right) \mathcal{D}\left(\begin{array}{c}
v \phi \\
\frac{1}{e} a^{\mu}
\end{array}\right) .
$$

Here, the matrix $\mathcal{D}$ in momentum space, which corresponds to the inverse propagator, is given by

$$
\begin{aligned}
\mathcal{D}(p) & =\left(\begin{array}{cc}
-p^{2} & \mathrm{i} \frac{\Delta_{B}}{2 B_{z}} \epsilon_{\nu \rho \sigma \tau}\left\langle f^{\sigma \tau}\right\rangle p^{\rho} \\
-\mathrm{i} \frac{\Delta_{B}}{2 B_{z}} \epsilon_{\mu \rho \sigma \tau}\left\langle f^{\sigma \tau}\right\rangle p^{\rho} & -\eta_{\mu \nu} p^{2}
\end{array}\right) \\
& \equiv\left(\begin{array}{cc}
-p^{2} & \boldsymbol{b}^{\dagger} \\
\boldsymbol{b} & -\eta_{\mu \nu} p^{2}
\end{array}\right),
\end{aligned}
$$

where $\boldsymbol{b}=\left(\mathrm{i} \Delta_{B} p^{z}, 0,0,-\mathrm{i} \Delta_{B} \omega\right)^{T}$ and we used the Feynman gauge. As we are interested in the static Wilson loop in the infrared (IR) regime, we consider the propagator at $\omega=0$,

$$
\mathcal{D}^{-1}(\boldsymbol{p})=\frac{1}{\boldsymbol{p}^{4}+\Delta_{B}^{2} p_{z}^{2}}\left(\begin{array}{ccc}
-\boldsymbol{p}^{2} & -\mathrm{i} \Delta_{B} p_{z} & \mathbf{0}^{T} \\
\mathrm{i} \Delta_{B} p_{z} & \boldsymbol{p}^{2} & \mathbf{0}^{T} \\
\mathbf{0} & \mathbf{0} & -\delta_{i j} \frac{\boldsymbol{p}^{4}+\Delta_{B}^{2} p_{z}^{2}}{\boldsymbol{p}^{2}}
\end{array}\right) .
$$

\footnotetext{
${ }^{8}$ In relativistic systems, the $\mathrm{U}(1)$ two-form symmetry cannot be broken spontaneously in $(3+1)$ dimensions due to the higherform generalization [17,59] of the Coleman-Mermin-WagnerHohenberg theorem [60-62]. In nonrelativistic systems, this theorem may be relaxed due to the modification of the propagator of a NG mode [63]. It is an open question whether the ColemanMermin-Wagner-Hohenberg theorem for higher-form symmetries can also be relaxed in nonrelativistic systems.
}

The nontrivial eigenvalues of the matrix due to $B_{z}$ are $\pm\left(\boldsymbol{p}^{4}+\Delta_{B}^{2} p_{z}^{2}\right)^{-1 / 2}$. Among them, the eigenvalue $\left(\boldsymbol{p}^{4}+\Delta_{B}^{2} p_{z}^{2}\right)^{-1 / 2}$ corresponds to an unphysical mode and we discard it. ${ }^{9}$

The potential $V(\boldsymbol{x})$ due to this mode in the IR limit is given by the inverse Fourier transformation of the propagator at $\omega=0$,

$\lim _{|\boldsymbol{x}| \rightarrow \infty} V(\boldsymbol{x}) \propto \lim _{|\boldsymbol{x}| \rightarrow \infty} \int \frac{\mathrm{d}^{3} \boldsymbol{p}}{(2 \pi)^{3}} \frac{1}{\left(\boldsymbol{p}^{4}+\Delta_{B}^{2} p_{z}^{2}\right)^{1 / 2}} \mathrm{e}^{\mathrm{i} \boldsymbol{p} \cdot \boldsymbol{x}}=0$,

and hence, the VEV of the Wilson loop is

$$
\langle W\rangle \sim \lim _{|\boldsymbol{x}| \rightarrow \infty} \mathrm{e}^{-T V(\boldsymbol{x})} \neq 0 .
$$

Therefore, the electric $\mathbb{Z}_{k}$ one-form symmetry is broken spontaneously.

We can also show the spontaneous breaking of the electric one-form symmetry in the case with the spatially varying axion background field. In this case, the matrix in Eq. (106) can be written as

$$
\mathcal{D}(p)=\left(\begin{array}{cc}
-p^{2} & 0 \\
0 & -\eta_{\mu \nu} p^{2}-\mathrm{i} \Delta_{\phi} \epsilon^{3 \mu \rho \nu} p_{\rho}
\end{array}\right) .
$$

In the static limit $\omega=0$, the nontrivial eigenvalues of the propagator due to $\left\langle\partial_{z} \phi\right\rangle$ is $-\left(\boldsymbol{p}^{4}+\Delta_{\phi}^{2} \boldsymbol{p}_{\perp}^{2}\right)^{-1 / 2}$. Here we discarded the eigenvalue $\left(\boldsymbol{p}^{4}+\Delta_{\phi}^{2} \boldsymbol{p}_{\perp}^{2}\right)^{-1 / 2}$ that corresponds to an unphysical mode. Similar to the argument above, the potential due to this mode in the limit $|\boldsymbol{x}| \rightarrow \infty$ vanishes, and thus, the electric $\mathbb{Z}_{k}$ one-form symmetry is broken spontaneously.

\section{Low-energy effective theory and chiral anomaly matching}

Since the system has gapped modes with the mass gap $\Delta_{B, \phi}$, one can derive a low-energy effective theory for gapless modes well below $\Delta_{B, \phi}$ by integrating out these gapped modes. One expects that the chiral anomaly in Eq. (59) should also be encoded in such a low-energy effective theory, since it is invariant under the renormalization. This is the 't Hooft anomaly matching. In the case of the spatially varying axion field, this is satisfied simply by

\footnotetext{
${ }^{9}$ In order to understand the fact that the eigenstate associated with the eigenvalue $\left(\boldsymbol{p}^{4}+\Delta_{B}^{2} p_{z}^{2}\right)^{-1 / 2}$ is unphysical, it is convenient to consider the case without the external magnetic field, where $\mathcal{D}=\operatorname{diag}\left(-p^{2}, p^{2},-p^{2},-p^{2},-p^{2}\right)$. In this case, one of the eigenvalues of $\mathcal{D}^{-1},-\frac{1}{\omega^{2}-p^{2}}$, which reduces to $\frac{1}{p^{2}}$ when $\omega=0$, corresponds to an unphysical ghost mode due to the gauge-fixing term. In our system, the eigenvalue $\left(\boldsymbol{p}^{4}+\Delta_{B}^{2} p_{z}^{2}\right)^{-1 / 2}$ corresponds to this unphysical mode, since it becomes $\frac{1}{p^{2}}$ when $\boldsymbol{B}=\mathbf{0}$.
} 
$\phi$ with the linear dispersion relation. On the other hand, in the case of the background magnetic field, the chiral anomaly matching is satisfied by the gapless mode with the quadratic dispersion relation (63), as we will show below.

Let us consider the case with the homogeneous background magnetic field. We define complex scalar fields ${ }^{10}$

$$
\begin{gathered}
\psi \equiv \sqrt{\frac{\Delta_{B}}{2}} \mathrm{e}^{\mathrm{i} \Delta_{B} t}\left(v \phi+\mathrm{i} \frac{a_{z}}{e}\right), \\
\psi^{*} \equiv \sqrt{\frac{\Delta_{B}}{2}} \mathrm{e}^{-\mathrm{i} \Delta_{B} t}\left(v \phi-\mathrm{i} \frac{a_{z}}{e}\right),
\end{gathered}
$$

from which we can write

$$
\begin{aligned}
& v \phi=\frac{1}{\sqrt{2 \Delta_{B}}}\left(\mathrm{e}^{-\mathrm{i} \Delta_{B} t} \psi+\mathrm{e}^{\mathrm{i} \Delta_{B} t} \psi^{*}\right), \\
& \frac{a_{z}}{e}=-\frac{\mathrm{i}}{\sqrt{2 \Delta_{B}}}\left(\mathrm{e}^{-\mathrm{i} \Delta_{B} t} \psi-\mathrm{e}^{\mathrm{i} \Delta_{B} t} \psi^{*}\right) .
\end{aligned}
$$

Inserting these relations into the original Lagrangian (58) and retaining the terms at the leading order in derivatives under the counting $\partial_{t} \sim \partial_{z} \sim \nabla_{\perp}^{2}$, where $\boldsymbol{\nabla}_{\perp} \equiv\left(\partial_{x}, \partial_{y}, 0\right)$, we derive the low-energy effective theory to the second order in fields,

$$
\begin{aligned}
\mathcal{L}_{\mathrm{EFT}}= & \frac{\mathrm{i}}{2} \psi^{*} \stackrel{\leftrightarrow}{\partial_{t}} \psi-\frac{1}{\Delta_{B}} \nabla_{\perp} \psi^{*} \cdot \nabla_{\perp} \psi+\frac{\sqrt{2 \Delta_{B}}}{e} \partial_{z} a^{t} \operatorname{Re}\left(\mathrm{e}^{-\mathrm{i} \Delta_{B} t} \psi\right) \\
& +\frac{1}{e} \sqrt{\frac{2}{\Delta_{B}}} \partial_{z} a_{\perp}^{\mu} \partial_{\mu} \operatorname{Im}\left(\mathrm{e}^{-\mathrm{i} \Delta_{B} t} \psi\right)-\frac{1}{4 e^{2}}\left|f_{\mu \nu}^{\perp}\right|^{2},
\end{aligned}
$$

up to total derivative terms, where $\stackrel{\leftrightarrow}{\partial_{t}} \equiv \vec{\partial}_{t}-\overleftarrow{\partial_{t}}, \quad V_{\perp}^{\mu} \equiv$ $\left(V^{t}, V^{x}, V^{y}, 0\right)$ for a generic vector $V^{\mu}$, and $f_{\mu \nu}^{\perp} \equiv \partial_{\mu} a_{\nu}^{\perp}-$ $\partial_{\nu} a_{\mu}^{\perp}$. It is clear that we have only gapless modes in this low-energy effective theory. In particular, the gapless mode $\psi$ has the quadratic dispersion relation (63) in the transverse direction as it should.

We note that the number of physical degrees of freedom in this effective theory decreases by one (which corresponds to the gapped mode) compared with that in the original theory (58). This can be understood from the fact that the number of physical degrees of freedom is equal to the number of real fields when the kinetic term is second order in time derivatives, while it is equal to the number of complex fields (or half of the number of real fields) when the kinetic term is first order in time derivatives [66].

\footnotetext{
${ }^{10}$ This procedure is similar to the one used to derive the nonrelativistic limit of the relativistic field theory for real scalar fields; see, e.g., Refs. [64-66].
}

The symmetries of this effective theory that we are interested in are as follows ${ }^{11}$ :

(i) Gauge symmetry

$$
\begin{aligned}
a_{\mu}^{\perp} & \rightarrow a_{\mu}^{\perp}+\partial_{\mu}^{\perp} \lambda, \\
\operatorname{Im}\left(\mathrm{e}^{-\mathrm{i} \Delta_{B} t} \psi\right) & \rightarrow \operatorname{Im}\left(\mathrm{e}^{-\mathrm{i} \Delta_{B} t} \psi\right)+\frac{1}{e} \sqrt{\frac{\Delta_{B}}{2}} \partial_{z} \lambda,
\end{aligned}
$$

with $\lambda$ a zero-form gauge parameter, which originates from the gauge symmetry $a_{\mu} \rightarrow a_{\mu}+\partial_{\mu} \lambda$ in the original theory (58).

(ii) Anomalous shift symmetry

$$
\operatorname{Re}\left(\mathrm{e}^{-\mathrm{i} \Delta_{B} t} \psi\right) \rightarrow \operatorname{Re}\left(\mathrm{e}^{-\mathrm{i} \Delta_{B} t} \psi\right)+\sqrt{\frac{\Delta_{B}}{2}} v c,
$$

with $c$ a constant, which originates from the anomalous shift symmetry $\phi \rightarrow \phi+c$ in the original theory (58).

In this effective theory, the canonical momentum of $\psi$ is

$$
\pi \equiv \frac{\partial \mathcal{L}_{\mathrm{EFT}}}{\partial\left(\partial_{t} \psi\right)}=\frac{\mathrm{i}}{2} \psi^{*}
$$

From the canonical commutation relation $[\psi(\boldsymbol{x}), \pi(\boldsymbol{y})]=$ $\frac{\mathrm{i}}{2} \delta(\boldsymbol{x}-\boldsymbol{y}),{ }^{12}$ we find

$$
\left[\psi(\boldsymbol{x}), \psi^{*}(\boldsymbol{y})\right]=\delta(\boldsymbol{x}-\boldsymbol{y}) .
$$

Alternatively, one can derive the commutation relation (118) by substituting Eq. (113) into the commutation relation in the original theory (58),

$$
v^{2}\left[\phi(\boldsymbol{x}), \partial_{t} \phi(\boldsymbol{y})\right]=\mathrm{i} \delta(\boldsymbol{x}-\boldsymbol{y}),
$$

and using the relation $v \partial_{t} \phi \approx \Delta_{B} \frac{a_{z}}{e}$ at low energy.

One way to check how the chiral anomaly is satisfied in this effective theory is to look at the anomalous commutator between the vector charge $n$ and axial charge $n_{5}$. For this purpose, let us derive the expressions of $n$ and $n_{5}$ in terms of $\psi$. The vector charge of the effective theory is given by

$$
n \equiv-\frac{\delta S_{\mathrm{EFT}}}{\delta a_{t}}=-\frac{\sqrt{2 \Delta_{B}}}{e} \partial_{z}\left[\operatorname{Re}\left(\mathrm{e}^{-\mathrm{i} \Delta_{B} t} \psi\right)\right]
$$

On the other hand, the axial charge in the theory (58) is $n_{5}=2 v^{2} \partial_{t} \phi$, which, in terms of $\psi$ reads

\footnotetext{
${ }^{11}$ We will not discuss the higher-form symmetries of the theory (114) because, as we only focus on the kinetic terms, the higherform symmetries turn out to be enhanced compared to the full theory with the interaction terms. A systematic construction of the full effective field theory based on the symmetries is deferred to future work.

${ }^{12}$ The factor $1 / 2$ here is due to the two-sided derivative $\stackrel{\leftrightarrow}{\partial_{t}}$ in Eq. (114).
} 


$$
n_{5}=2 v \sqrt{\frac{2}{\Delta_{B}}} \partial_{t}\left[\operatorname{Re}\left(\mathrm{e}^{-\mathrm{i} \Delta_{B} t} \psi\right)\right] .
$$

By using Eq. (118), we arrive at

$$
\left[n(\boldsymbol{x}), n_{5}(\boldsymbol{y})\right]=-\mathrm{i} \frac{k}{2 \pi^{2}} B_{z} \partial_{z} \delta(\boldsymbol{x}-\boldsymbol{y}) .
$$

This is the anomalous commutator [67] that is responsible for the chiral anomaly, ${ }^{13}$ and the anomaly matching is correctly satisfied in this effective theory.

\section{DISCUSSIONS}

In this paper, we studied the axion electrodynamics with level $k$ in $(3+1)$ dimensions in background fields as a paradigmatic example of topological mass generation in gapless systems. We have shown that this system exhibits the spontaneous breaking of the $\mathbb{Z}_{k}$ one-form symmetry for $k \geq 2$ even in the absence of the conventional topological order.

Since the low-energy effective theory of QCD coupled to QED corresponds to the axion electrodynamics in Eq. (58) with $|k|=1$ as mentioned in the main text, one can ask whether the case with $|k| \geq 2$ can be realized in physical

\footnotetext{
${ }^{13}$ In Ref. [67], chiral/Dirac fermions with $|k|=1$ are considered. The anomalous commutator (122) with a generic integer $k$ appears in a system having a monopole with charge $k$ in momentum space that acts as a source/sink of Berry curvature [68]; see also Sec. IV.
}

systems. One such possibility is the "axionic charge density wave" (CDW) [69] in multi-Weyl semimetals. The multiWeyl semimetal $[70,71]$ is a type of Weyl semimetal in which pairs of monopoles and antimonopoles with a generic integer charge $k$ appear in momentum space and where the chiral anomaly relation (59) is realized. In Weyl semimetals, the interaction effect may lead to the dynamical chiral symmetry breaking by the pairing between electrons and holes with opposite chiralities and, consequently, a NG mode $\phi$, which may be regarded as an "axion" field, appears [69]. It has been recently reported that evidence of such an axionic CDW is experimentally observed in the Weyl semimetal $\left(\mathrm{TaSe}_{4}\right)_{2} \mathrm{I}$ for $|k|=1$ [72]. If the axion CDW is realized in multi-Weyl semimetals with $|k| \geq 2$, the effective theory for the NG mode $\phi$ coupled to dynamical electromagnetic fields at low energy there is the axion electrodynamics with level $k$.

In this paper, we also constructed the low-energy effective theory (114) for the gapless mode with the quadratic dispersion relation, starting from the theory (58) in the external magnetic field by integrating out the gapped modes. It would be interesting to develop a systematic construction of this kind of low-energy effective theory based on the breaking pattern of zero- and higher-form symmetries without referring to the details of a microscopic theory.

\section{ACKNOWLEDGMENTS}

R. Y. thanks Yoshimasa Hidaka and Noriyuki Sogabe for discussions. N. Y. was supported by Keio Institute of Pure and Applied Sciences (KiPAS) project at Keio University and JSPS KAKENHI Grant No. 19K03852.
[1] E.C.G. Stueckelberg, Theory of the radiation of photons of small arbitrary mass, Helv. Phys. Acta 30, 209 (1957).

[2] N. Yamamoto, Axion electrodynamics and nonrelativistic photons in nuclear and quark matter, Phys. Rev. D 93, 085036 (2016).

[3] S. Ozaki and N. Yamamoto, Axion crystals, J. High Energy Phys. 08 (2017) 098.

[4] N. Sogabe and N. Yamamoto, Triangle anomalies and nonrelativistic Nambu-goldstone modes of generalized global symmetries, Phys. Rev. D 99, 125003 (2019).

[5] T. Brauner and S. V. Kadam, Anomalous low-temperature thermodynamics of QCD in strong magnetic fields, J. High Energy Phys. 11 (2017) 103.

[6] J. M. Maldacena, G. W. Moore, and N. Seiberg, D-brane charges in five-brane backgrounds, J. High Energy Phys. 10 (2001) 005.

[7] G. T. Horowitz, Exactly soluble diffeomorphism invariant theories, Commun. Math. Phys. 125, 417 (1989).
[8] M. Blau and G. Thompson, A new class of topological field theories and the Ray-Singer torsion, Phys. Lett. B 228, 64 (1989).

[9] T. J. Allen, M. J. Bowick, and A. Lahiri, Topological mass generation in $(3+1)$-dimensions, Mod. Phys. Lett. A 06, 559 (1991).

[10] E. Cremmer and J. Scherk, Spontaneous dynamical breaking of gauge symmetry in dual models, Nucl. Phys. B72, 117 (1974).

[11] R. L. Davis and E. P. S. Shellard, Antisymmetric tensors and spontaneous symmetry breaking, Phys. Lett. B 214, 219 (1988).

[12] B. Binegar, Relativistic field theories in three-dimensions, J. Math. Phys. (N.Y.) 23, 1511 (1982).

[13] S. Deser, R. Jackiw, and S. Templeton, Three-Dimensional Massive Gauge Theories, Phys. Rev. Lett. 48, 975 (1982).

[14] S. Deser, R. Jackiw, and S. Templeton, Topologically massive gauge theories, Ann. Phys. (N.Y.) 140, 372 (1982); 281, 409 (2000). 
[15] E. Witten, Instantons, the quark model, and the $1 / \mathrm{n}$ expansion, Nucl. Phys. B149, 285 (1979).

[16] A. Aurilia, Y. Takahashi, and P. K. Townsend, The U(1) problem and the Higgs mechanism in two-dimensions and four-dimensions, Phys. Lett. 95B, 265 (1980).

[17] D. Gaiotto, A. Kapustin, N. Seiberg, and B. Willett, Generalized global symmetries, J. High Energy Phys. 02 (2015) 172.

[18] C. D. Batista and Z. Nussinov, Generalized Elitzur's theorem and dimensional reduction, Phys. Rev. B 72, 045137 (2005).

[19] T. Pantev and E. Sharpe, GLSM's for Gerbes (and other toric stacks), Adv. Theor. Math. Phys. 10, 77 (2006).

[20] T. Pantev and E. Sharpe, String compactifications on CalabiYau stacks, Nucl. Phys. B733, 233 (2006).

[21] T. Pantev and E. Sharpe, Notes on gauging noneffective group actions, arXiv:hep-th/0502027.

[22] Z. Nussinov and G. Ortiz, Sufficient symmetry conditions for topological quantum order, Proc. Natl. Acad. Sci. U.S.A. 106, 16944 (2009).

[23] Z. Nussinov and G. Ortiz, Autocorrelations and thermal fragility of anyonic loops in topologically quantum ordered systems, Phys. Rev. B 77, 064302 (2008).

[24] Z. Nussinov and G. Ortiz, A symmetry principle for topological quantum order, Ann. Phys. (Amsterdam) 324, 977 (2009).

[25] T. Banks and N. Seiberg, Symmetries and strings in field theory and gravity, Phys. Rev. D 83, 084019 (2011).

[26] J. Distler and E. Sharpe, Quantization of Fayet-Iliopoulos parameters in supergravity, Phys. Rev. D 83, 085010 (2011).

[27] Z. Nussinov, G. Ortiz, and E. Cobanera, Effective and exact holographies from symmetries and dualities, Ann. Phys. (Amsterdam) 327, 2491 (2012).

[28] A. Kapustin and N. Seiberg, Coupling a QFT to a TQFT and duality, J. High Energy Phys. 04 (2014) 001.

[29] Y. Hidaka, Y. Hirono, and R. Yokokura, Counting NambuGoldstone Modes of Higher-Form Global Symmetries, Phys. Rev. Lett. 126, 071601 (2021).

[30] X. G. Wen, Vacuum degeneracy of chiral spin states in compactified space, Phys. Rev. B 40, 7387 (1989).

[31] X. G. Wen, Topological order in rigid states, Int. J. Mod. Phys. B 04, 239 (1990).

[32] T. H. Hansson, V. Oganesyan, and S. L. Sondhi, Superconductors are topologically ordered, Ann. Phys. (Amsterdam) 313, 497 (2004).

[33] H. B. Nielsen and S. Chadha, On how to count Goldstone bosons, Nucl. Phys. B105, 445 (1976).

[34] H. Watanabe and T. Brauner, On the number of NambuGoldstone bosons and its relation to charge densities, Phys. Rev. D 84, 125013 (2011).

[35] H. Watanabe and H. Murayama, Unified Description of Nambu-Goldstone Bosons without Lorentz in Variance, Phys. Rev. Lett. 108, 251602 (2012).

[36] Y. Hidaka, Counting Rule for Nambu-Goldstone Modes in Nonrelativistic Systems, Phys. Rev. Lett. 110, 091601 (2013).

[37] G. T. Horowitz and M. Srednicki, A quantum field theoretic description of linking numbers and their generalization, Commun. Math. Phys. 130, 83 (1990).

[38] A. A. Abrikosov, On the Magnetic properties of superconductors of the second group, Sov. Phys. JETP 5, 1174
(1957), http://www.jetp.ac.ru/cgi-bin/e/index/e/5/6/p1174? $\mathrm{a}=$ list.

[39] H. B. Nielsen and P. Olesen, Vortex line models for dual strings, Nucl. Phys. B61, 45 (1973).

[40] I. Oda and S. Yahikozawa, Linking numbers and variational method, Phys. Lett. B 238, 272 (1990).

[41] X. Chen, A. Tiwari, and S. Ryu, Bulk-boundary correspondence in $(3+1)$-dimensional topological phases, Phys. Rev. B 94, 045113 (2016); 94, 079903 (2016).

[42] Y. Hidaka, Y. Hirono, M. Nitta, Y. Tanizaki, and R. Yokokura, Topological order in the color-flavor locked phase of a $(3+1)$-dimensional $\mathrm{U}(\mathrm{N})$ gauge-Higgs system, Phys. Rev. D 100, 125016 (2019).

[43] K.-M. Lee, The dual formulation of cosmic strings and vortices, Phys. Rev. D 48, 2493 (1993).

[44] M. M. Anber and E. Poppitz, Anomaly matching, (axial) Schwinger models, and high-T super Yang-Mills domain walls, J. High Energy Phys. 09 (2018) 076.

[45] A. Armoni and S. Sugimoto, Vacuum structure of charge $\mathrm{k}$ two-dimensional QED and dynamics of an anti D-string near an $\mathrm{O}^{-}$-plane, J. High Energy Phys. 03 (2019) 175.

[46] T. Misumi, Y. Tanizaki, and M. Ünsal, Fractional $\theta$ angle, 't Hooft anomaly, and quantum instantons in charge- $q$ multiflavor Schwinger model, J. High Energy Phys. 07 (2019) 018.

[47] J. D. Brown and C. Teitelboim, Dynamical neutralization of the cosmological constant, Phys. Lett. B 195, 177 (1987).

[48] J. D. Brown and C. Teitelboim, Neutralization of the cosmological constant by membrane creation, Nucl. Phys. B297, 787 (1988).

[49] M. J. Duff, The cosmological constant is possibly zero, but the proof is probably wrong, Phys. Lett. B 226, 36 (1989).

[50] M. J. Duncan and L. G. Jensen, Four forms and the vanishing of the cosmological constant, Nucl. Phys. B336, 100 (1990).

[51] M. J. Duncan and L. G. Jensen, A new mechanism for neutralizing the cosmological constant, Nucl. Phys. B361, 695 (1991).

[52] N. Kaloper and L. Sorbo, A Natural Framework for Chaotic Inflation, Phys. Rev. Lett. 102, 121301 (2009).

[53] N. Kaloper and L. Sorbo, Where in the string landscape is quintessence, Phys. Rev. D 79, 043528 (2009).

[54] E. Sharpe, Notes on generalized global symmetries in QFT, Fortschr. Phys. 63, 659 (2015).

[55] Y. Hidaka, M. Nitta, and R. Yokokura, Emergent discrete 3-form symmetry and domain walls, Phys. Lett. B 803, 135290 (2020).

[56] Y. Hidaka, M. Nitta, and R. Yokokura, Higher-form symmetries and 3-group in axion electrodynamics, Phys. Lett. B 808, 135672 (2020).

[57] R. Dijkgraaf and E. Witten, Topological gauge theories and group cohomology, Commun. Math. Phys. 129, 393 (1990).

[58] Y. Hidaka, M. Nitta, and R. Yokokura, Global 3-group symmetry and 't Hooft anomalies in axion electrodynamics, J. High Energy Phys. 01 (2021) 173.

[59] E. Lake, Higher-form symmetries and spontaneous symmetry breaking, arXiv:1802.07747.

[60] S. R. Coleman, There are no Goldstone bosons in twodimensions, Commun. Math. Phys. 31, 259 (1973). 
[61] N. D. Mermin and H. Wagner, Absence of Ferromagnetism or Antiferromagnetism in One-Dimensional or Two-Dimensional Isotropic Heisenberg Models, Phys. Rev. Lett. 17, 1133 (1966).

[62] P. C. Hohenberg, Existence of long-range order in one and two dimensions, Phys. Rev. 158, 383 (1967).

[63] H. Watanabe, Counting rules of Nambu-Goldstone modes, Annu. Rev. Condens. Matter Phys. 11, 169 (2020).

[64] A. H. Guth, M. P. Hertzberg, and C. Prescod-Weinstein, Do dark matter axions form a condensate with long-range correlation?, Phys. Rev. D 92, 103513 (2015).

[65] M. H. Namjoo, A. H. Guth, and D. I. Kaiser, Relativistic corrections to nonrelativistic effective field theories, Phys. Rev. D 98, 016011 (2018).

[66] E. Braaten, A. Mohapatra, and H. Zhang, Classical nonrelativistic effective field theories for a real scalar field, Phys. Rev. D 98, 096012 (2018).
[67] S. L. Adler and D. Boulware, Anomalous commutators and the triangle diagram, Phys. Rev. 184, 1740 (1969).

[68] D. T. Son and N. Yamamoto, Berry Curvature, Triangle Anomalies, and the Chiral Magnetic Effect in Fermi Liquids, Phys. Rev. Lett. 109, 181602 (2012).

[69] Z. Wang and S.-C. Zhang, Chiral anomaly, charge density waves, and axion strings from Weyl semimetals, Phys. Rev. B 87, 161107 (2013).

[70] G. Xu, H. Weng, Z. Wang, X. Dai, and Z. Fang, Chern Semimetal and the Quantized Anomalous Hall Effect in $\mathrm{HgCr}_{2} \mathrm{Se}_{4}$, Phys. Rev. Lett. 107, 186806 (2011).

[71] C. Fang, M. J. Gilbert, X. Dai, and B. A. Bernevig, Multi-Weyl Topological Semimetals Stabilized by Point Group Symmetry, Phys. Rev. Lett. 108, 266802 (2012).

[72] J. Gooth et al., Axionic charge-density wave in the Weyl semimetal $\left(\mathrm{TaSe}_{4}\right)_{2} \mathrm{I}$, arXiv:1906.04510. 